Article

\title{
Timoshenko Beam Theory: Exact Solution for First-Order Analysis, Second-Order
}

\section{Analysis, and Stability Using a Moment-Shear Force-Curvature Relationship}

\author{
Valentin Fogang \\ Civil Engineer, C/o BUNS Sarl, P.O Box 1130, Yaounde, Cameroon; valentin.fogang@bunscameroun.com
}

ORCID iD https://orcid.org/0000-0003-1256-9862

\begin{abstract}
This paper presents an exact solution to the Timoshenko beam theory (TBT) for first-order analysis, secondorder analysis, and stability. The TBT covers cases associated with small deflections based on shear deformation considerations, whereas the Euler-Bernoulli beam theory (EBBT) neglects shear deformations. Thus, the EulerBernoulli beam is a special case of the Timoshenko beam. The moment-curvature relationship is one of the governing equations of the EBBT, and closed-form expressions of efforts and deformations are available in the literature. However, neither an equivalent to the moment-curvature relationship of EBBT nor closed-form expressions of efforts and deformations can be found in the TBT. In this paper, a moment-shear force-curvature relationship, the equivalent in TBT of the moment-curvature relationship of EBBT, was presented. Based on this relationship, first-order and second-order analyses were conducted, and closed-form expressions of efforts and deformations were derived for various load cases. Furthermore, beam stability was analyzed and buckling loads were calculated. Finally, first-order and second-order element stiffness matrices were determined.
\end{abstract}

Keywords: Timoshenko beam; moment-shear force-curvature relationship; closed-form solutions; non-uniform heating; stability; second-order element stiffness matrix; elastic Winkler foundation

\section{Introduction}

The Timoshenko beam theory (TBT) was developed by Stephen Timoshenko (1921) early in the $20^{\text {th }}$ century. The model accounts for shear deformations, making it suitable for describing the behavior of thick beams, whereas the Euler-Bernoulli beam theory (EBBT) neglects them. If the shear modulus of the beam material approaches infinity, the 


\section{TIMOSHENKO BEAM THEORY EXACT SOLUTION}

beam becomes rigid in shear and the TBT converges towards EBBT. Thus, the Euler-Bernoulli beam is a special case of the Timoshenko beam. First-order analysis of the Timoshenko beam is routinely performed; the principle of virtual work yields accurate results and is easy to apply. Unfortunately, second-order analysis of the Timoshenko beam cannot be modeled with the principle of virtual work. Various studies have focused on the analysis of Timoshenko beams, most of which using numerical methods. Sang-Ho et al. (2019) presented a nonlinear finite element analysis formulation for shear in reinforced concrete Beams; that formulation utilizes an equilibrium-driven shear stress function. Abbas and Mohammad (2013) suggested a two-node finite element for analyzing the stability and free vibration of Timoshenko beam; interpolation functions for displacement field and beam rotation were exactly calculated by employing total beam energy and its stationing to shear strain. Hayrullah and Mustapha (2017) performed a buckling analysis of a nano sized beam by using Timoshenko beam theory and Eringen's nonlocal elasticity theory; the vertical displacement function and the rotation function are chosen as Fourier series. Chen et al. (2018) used the variational iteration method to analyze the flexural vibration of rotating Timoshenko beams; accurate natural frequencies and mode shapes under various rotation speeds and rotary inertia were obtained. Onyia and Rowland-Lato (2018) presented a finite element formulation for the determination of the critical buckling load of unified beam element that is free from shear locking using the energy method; the proposed technique provides a unified approach for the stability analysis of beams with any end conditions. Pavlovic and Pavlović (2018) investigated the dynamic stability problem of a Timoshenko beam supported by a generalized Pasternak-type viscoelastic foundation subjected to compressive axial loading, where rotary inertia is neglected; the direct Liapunov method was used. In stability analysis Timoshenko and Gere (1961) proposed formulas to account for shear stiffness by means of calculation of buckling loads of the associated Euler-Bernoulli beams. Hu et al. (2019) used matrix structural analysis to derive a closed-form solution of the secondorder element stiffness matrix; the buckling loads of single-span beams were also determined.

The moment-curvature relationship, one of the governing equations of the EBBT, has no equivalent in the TBT literature. Furthermore, closed-form expressions of efforts and deformations are not common. In this paper a moment-shear force-curvature relationship (MSFCR), the equivalent in TBT of the moment-curvature relationship of EBBT, was presented. The relationship between the curvature, the bending moment, the bending stiffness, the shear force, and the shear stiffness was then described. Based on MSFCR, closed-form expressions of efforts and 


\section{TIMOSHENKO BEAM THEORY EXACT SOLUTION}

deformations were derived, as well as first-order and second-order element stiffness matrices. Stability analysis was conducted, and the buckling lengths of single-span systems were determined.

\section{Materials and Methods}

\subsection{Governing equations}

\subsubsection{Statics}

The sign convention adopted for the loads, bending moments, shear forces, and displacements is illustrated in Figure 1.

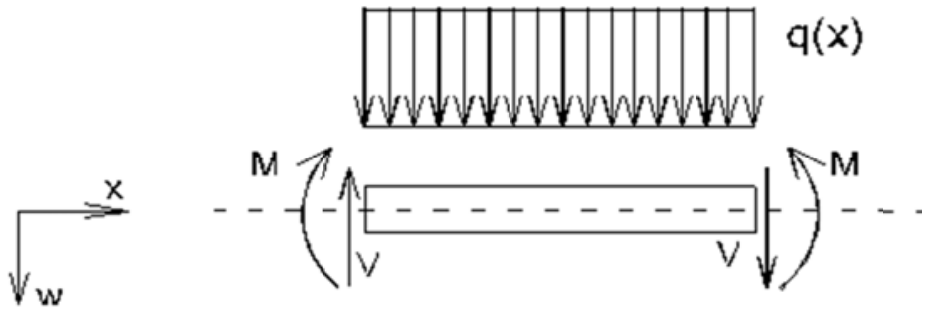

Fig 1 Sign convention for loads, bending moments, shear forces, and displacements

Specifically, $\mathrm{M}(x)$ is the bending moment in the section, $\mathrm{V}(x)$ is the shear force, $\mathrm{w}(x)$ is the deflection, and $\mathrm{q}(x)$ is the distributed load in the positive downward direction.

In first-order analysis the equations of static equilibrium on an infinitesimal element are as follows:

$$
V(x)=\frac{d M(x)}{d x} \text { (1) } \quad \frac{d^{2} M(x)}{d x^{2}}=-q(x)
$$

According to the Timoshenko beam theory, the bending moment and the shear force are related to the deflection $\mathrm{w}(x)$ and the rotation (positive in clockwise) of cross section $\varphi(\mathrm{x})$ as follows:

$$
M(x)=-E I \frac{d \varphi(x)}{d x} \quad \text { (3) } \quad V(x)=\kappa G A \times\left(\frac{d w(x)}{d x}-\varphi(x)\right)
$$

In these equations $\mathrm{E}$ is the elastic modulus, $\mathrm{I}$ is the second moment of area, $\kappa$ is the shear correction factor, $\mathrm{G}$ is the shear modulus, and A is the cross section area. Equations (1), (3), and (4) also apply in second-order analysis.

\subsubsection{Material and geometric equations}

Equation (4) can be formulated as follows

$$
\frac{d w(x)}{d x}=\varphi(x)+\frac{V(x)}{\kappa G A}
$$


Differentiating both sides of Equation (5) with respect to $x$ yields the following equation:

$$
\frac{d^{2} w(x)}{d x^{2}}=\frac{d \varphi(x)}{d x}+\frac{d}{d x}\left(\frac{V(x)}{\kappa G A}\right)
$$

Substituting Equation (3) into Equation (6) yields the following:

$$
\frac{d^{2} w(x)}{d x^{2}}=-\frac{M(x)}{E I}+\frac{d}{d x}\left(\frac{V(x)}{\kappa G A}\right)
$$

Equation (7) yields the following moment-shear force-curvature relationship (MSFCR) combining bending and shear:

$$
\frac{d^{2} w(x)}{d x^{2}}+\frac{M(x)}{E I}-\frac{d}{d x}\left(\frac{V(x)}{\kappa G A}\right)=0
$$

For a uniform beam along segments, substituting Equation (1) into Equation (8) yields,

$$
\frac{d^{2} w(x)}{d x^{2}}+\frac{M(x)}{E I}-\frac{1}{\kappa G A} \frac{d^{2} M(x)}{d x^{2}}=0
$$

For a tapered beam, substituting Equation (1) into Equation (8) yields,

$$
\frac{d^{2} w(x)}{d x^{2}}+\frac{M(x)}{E I(x)}-\frac{1}{\kappa G A(x)} \frac{d^{2} M(x)}{d x^{2}}+\frac{1}{(\kappa G A(x))^{2}} \times \frac{d \kappa G A(x)}{d x} \times \frac{d M(x)}{d x}=0
$$

In the case of non-uniform heating, Equation (9) becomes:

$$
\frac{d^{2} w(x)}{d x^{2}}+\frac{M(x)}{E I}-\frac{1}{\kappa G A} \times \frac{d^{2} M(x)}{d x^{2}}+\frac{\alpha_{T} \Delta T}{d}=0,
$$

where $\alpha_{\mathrm{T}}$ is the coefficient of thermal expansion, $\Delta \mathrm{T}=\Delta \mathrm{T}_{\mathrm{bb}}-\Delta \mathrm{T}_{\mathrm{tb}}$ is the difference between the temperature changes at the beam's bottom fibers $\left(\Delta \mathrm{T}_{\mathrm{bb}}\right)$ and top fibers $\left(\Delta \mathrm{T}_{\mathrm{tb}}\right)$, and $\mathrm{d}$ is the height of the beam.

Combining Equations (1) and (5) yields the rotation angle as follows:

$$
\varphi(x)=\frac{d w(x)}{d x}-\frac{1}{\kappa G A} \frac{d M(x)}{d x}
$$

Equations (5) to (12) apply as well in first-order analysis and in second-order analysis.

\subsubsection{Summary of Timoshenko and Euler-Bernoulli beam equations}

Table 1 summarizes the fundamental Timoshenko beam equations and compares them to the Euler-Bernoulli beam equations. 
Table 1. Summary of equations for Timoshenko and Euler-Bernoulli beams

\begin{tabular}{|c|c|c|}
\hline & Timoshenko beam & Euler-Bernoulli beam \\
\hline Statics & $V(x)=\frac{d M(x)}{d x}$ & $V(x)=\frac{d M(x)}{d x}$ \\
\hline Geometry & $\varphi(x)=\frac{d w(x)}{d x}-\frac{1}{\kappa G A} \times \frac{d M(x)}{d x}$ & $\varphi(x)=\frac{d w(x)}{d x}$ \\
\hline $\begin{array}{c}\text { Moment-shear } \\
\text { force-curvature } \\
\text { equation }\end{array}$ & $\frac{d^{2} w(x)}{d x^{2}}+\frac{M(x)}{E I}-\frac{d}{d x}\left(\frac{V(x)}{\kappa G A}\right)=0$ & $\frac{d^{2} w(x)}{d x^{2}}+\frac{M(x)}{E I}=0$ \\
\hline
\end{tabular}

The bending shear factor is defined as follows:

$$
\alpha=\frac{E I}{l^{2} \kappa G A}
$$

\subsection{First-order analysis of uniform Timoshenko beams}

\subsubsection{Governing equations}

The application of Equation (2) yields the following formulation of the bending moment:

$$
M(x)=-\iint q(x) d x d x+C_{1} x+C_{2}
$$

Substituting Equation (2) into the MSFCR (Equation (9)) for a uniform beam and integrating twice yields:

$$
E I \times w(x)=-\iint\left[\frac{E I}{\kappa G A} q(x)+M(x)\right] d x d x+C_{3} x+C_{4}
$$

The shear forces and rotation angles are determined using Equations (1) and (14), and Equations (12), (14), and (15), respectively.

$$
\begin{aligned}
& V(x)=-\int q(x) d x+C_{1} \\
& E I \times \varphi(x)=-\int M(x) d x-\frac{E I}{\kappa G A} C_{1}+C_{3}
\end{aligned}
$$

The integration constants $\mathrm{C}_{\mathrm{i}}(\mathrm{i}=1,2,3$, and 4$)$ are determined using the boundary conditions and continuity equations and combining the deflections, the rotation angles, the bending moments, and the shear forces. 


\subsubsection{Analysis of some load cases}

Detailed results for various support conditions and loadings are presented in Appendix B.

\section{Uniformly distributed load $q$}

Applying Equations (14) to (15b) yields

$$
\begin{aligned}
& M(x)=-\frac{q}{2} x^{2}+C_{1} x+C_{2}, \quad V(x)=-q x+C_{1} \\
& E I \times w(x)=\frac{q}{24} x^{4}-\frac{C_{1}}{6} x^{3}-\frac{1}{2}\left(C_{2}+\alpha l^{2} q\right) x^{2}+C_{3} x+C_{4} \\
& E I \times \varphi(x)=\frac{q}{6} x^{3}-\frac{C_{1}}{2} x^{2}-C_{2} x-\alpha l^{2} C_{1}+C_{3}
\end{aligned}
$$

\section{Non-uniform heating:}

Equation (11) is applied instead of Equation (9). The thermal curvature strain is $\quad \kappa_{T}=\frac{\alpha_{T} \Delta T}{d}$.

Equation (15c) becomes

$$
\begin{aligned}
& M(x)=C_{1} x+C_{2}, \quad V(x)=C_{1} \\
& E I \times w(x)=-\frac{C_{1}}{6} x^{3}-\frac{1}{2}\left(C_{2}+E I \kappa_{T}\right) x^{2}+C_{3} x+C_{4} \\
& E I \times \varphi(x)=-\frac{C_{1}}{2} x^{2}-\left(C_{2}+E I \kappa_{T}\right) x+C_{3}-\alpha l^{2} C_{1}
\end{aligned}
$$

\subsubsection{Element stiffness matrix}

The sign convention for bending moments, shear forces, displacements, and rotation angles adopted for use in determining the element stiffness matrix in local coordinates is illustrated in Figure 2.

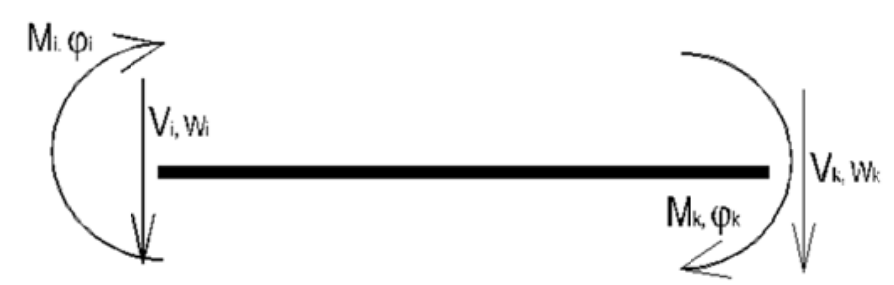

Fig 2 Sign convention for bending moments, shear forces, displacements, and rotation angles for first-order $4 \times 4$ element stiffness matrix 
Let us define following vectors,

$$
\vec{S}=\left[V_{i} ; M_{i} ; V_{k} ; M_{k}\right]^{T}
$$

$$
\vec{V}=\left[w_{i} ; \varphi_{i} ; w_{k} ; \varphi_{k}\right]^{T}
$$

The element stiffness matrix in local coordinates of the Timoshenko beam is denoted by $\mathrm{K}_{\mathrm{Tb}}$. The relationship between the aforementioned vectors is as follows:

$$
\vec{S}=K_{T b l} \times \vec{V}
$$

Applying Equation (15c) with the distributed load $\mathrm{q}=0$, yields the following:

$$
\begin{aligned}
& M(x)=C_{1} x+C_{2} \quad V(x)=C_{1} \\
& E I \times w(x)=-\frac{C_{1}}{6} x^{3}-\frac{C_{2}}{2} x^{2}+C_{3} x+C_{4} \\
& E I \times \varphi(x)=-\frac{C_{1}}{2} x^{2}-C_{2} x-\alpha l^{2} C_{1}+C_{3}
\end{aligned}
$$

Considering the sign conventions adopted for bending moments and shear forces in general (see Figure 1) and for bending moments and shear forces in the element stiffness matrix (see Figure 2), we can set following static compatibility boundary conditions using Equation (19):

$$
\begin{aligned}
& V_{i}=-V(x=0)=-C_{1} \\
& M_{i}=M(x=0)=C_{2} \\
& V_{k}=V(x=l)=C_{1} \\
& M_{k}=-M(x=l)=-C_{1} l-C_{2}
\end{aligned}
$$

Considering the sign conventions adopted for the displacements and rotations in general (see Figure 1) and for displacements and rotations in the member stiffness matrix (see Figure 2), we can set following geometric compatibility boundary conditions using Equations (20) and (20a):

$$
\begin{aligned}
& w(x=0)=w_{i} \rightarrow E I \times w_{i}=C_{4} \\
& \varphi(x=0)=\varphi_{i} \rightarrow E I \times \varphi_{i}=-d^{2} C_{1}+C_{3} \\
& w(x=l)=w_{k} \rightarrow E I \times w_{k}=l^{3} / 6 \times C_{1}-l^{2} / 2 \times C_{2}+l C_{3}+C_{4} \\
& \varphi(x=l)=\varphi_{k} \rightarrow E I \times \varphi_{k}=(-1 / 2-\alpha) \times l^{2} C_{1}-l C_{2}+C_{3}
\end{aligned}
$$


The combination of Equations (16) to (18) and Equations (21) to (28) yields the first-order element stiffness matrix of the Timoshenko beam:

$$
K_{T b l}=E I \times\left[\begin{array}{cccc}
-1 & 0 & 0 & 0 \\
0 & 1 & 0 & 0 \\
1 & 0 & 0 & 0 \\
-l & -1 & 0 & 0
\end{array}\right] \times\left[\begin{array}{cccc}
0 & 0 & 0 & 1 \\
-\alpha l^{2} & 0 & 1 & 0 \\
-l^{3} / 6 & -l^{2} / 2 & l & 1 \\
(-1 / 2-\alpha) l^{2} & -l & 1 & 0
\end{array}\right]^{-1}
$$

The development of Equation (28a) yields the following widely known formulation of the first-order element stiffness matrix of the Timoshenko beam:

$$
K_{T b l}=\left[\begin{array}{ccccc}
\frac{12}{1+\varphi} \frac{E I}{l^{3}} & \frac{6}{1+\varphi} \frac{E I}{l^{2}} & \frac{-12}{1+\varphi} \frac{E I}{l^{3}} & \frac{6}{1+\varphi} \frac{E I}{l^{2}} \\
& \frac{4+\varphi}{1+\varphi} \frac{E I}{l} & \frac{-6}{1+\varphi} \frac{E I}{l^{2}} & \frac{2-\varphi}{1+\varphi} \frac{E I}{l} \\
s y m & \frac{12}{1+\varphi} \frac{E I}{l^{3}} & \frac{-6}{1+\varphi} \frac{E I}{l^{2}} \\
& & \frac{4+\varphi}{1+\varphi} \frac{E I}{l}
\end{array}\right]
$$

where

$$
\varphi=\frac{12 E I}{l^{2} \kappa G A}
$$

Assuming the presence of a hinge at the right end, the sign convention for bending moments, shear forces, displacements, and rotations is illustrated in Figure 3.

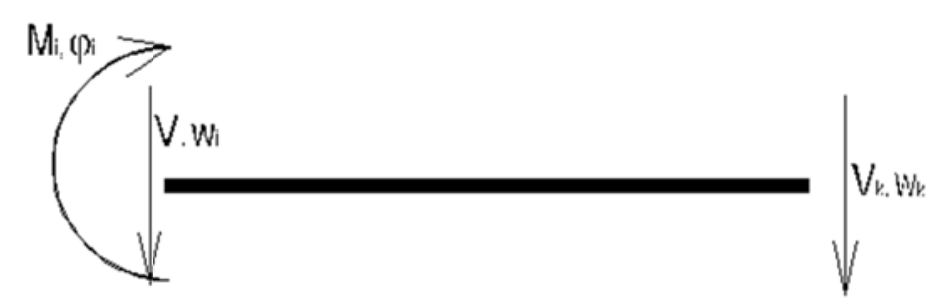

Fig 3 Sign conventions for bending moments, shear forces, displacements, and rotation angles for first-order $3 \times 3$ element stiffness matrix 
The vectors of Equations (16) and (17) become

$$
\vec{S}=\left[V_{i} ; M_{i} ; V_{k}\right]^{T} \quad(31) \quad \vec{V}=\left[w_{i} ; \varphi_{i} ; w_{k}\right]^{T}
$$

The element stiffness matrix becomes:

$$
K_{T b l}=\left[\begin{array}{cccc}
\frac{3}{1+\varphi^{\prime}} \frac{E I}{l^{3}} & \frac{3}{1+\varphi^{\prime}} \frac{E I}{l^{2}} & \frac{-3}{1+\varphi^{\prime}} \frac{E I}{l^{3}} \\
\frac{3}{1+\varphi^{\prime}} \frac{E I}{l} & \frac{-3}{1+\varphi^{\prime}} \frac{E I}{l^{2}} \\
s y m & \frac{3}{1+\varphi^{\prime}} \frac{E I}{l^{3}}
\end{array}\right]
$$

where

$$
\varphi^{\prime}=\frac{3 E I}{l^{2} \kappa G A}
$$

\subsubsection{Beam resting on an elastic Winkler foundation}

For a beam resting on a Winkler foundation with stiffness $\mathrm{K}_{\mathrm{w}}$, Equation (2) of static equilibrium becomes,

$$
\frac{d^{2} M(x)}{d x^{2}}-K_{w} w(x)=-q(x)(35) \quad K_{w}=k_{w} \frac{E I}{l^{4}}
$$

Differentiating Equation (35) twice with respect to $x$, combined with the MSFCR (Equation (9)), yields:

$$
\frac{d^{4} M(x)}{d x^{4}}-\frac{K_{w}}{\kappa G A} \frac{d^{2} M(x)}{d x^{2}}+\frac{K_{w}}{E I} M(x)=-\frac{d^{2} q(x)}{d x^{2}}
$$

The solution of Equation (36) yields the formulation of $M(x)$ with four integration constants $\left(A_{1}, B_{1}, C_{1}, D_{1}\right)$. The shape of $\mathrm{M}(\mathrm{x})$ depends on the parameter $\mathrm{k}_{\mathrm{W}}-4 / \alpha^{2}$ as follows:

$M(x)=A_{1} \cosh \xi_{1} \frac{x}{l}+B_{1} \sinh \xi_{1} \frac{x}{l}+C_{1} \cosh \xi_{2} \frac{x}{l}+D_{1} \sinh \xi_{2} \frac{x}{l}+M_{p}(x)$

$M(x)=A_{1} \cosh \xi_{1} \frac{x}{l}+B_{1} \xi_{1} \frac{x}{l} \cosh \xi_{1} \frac{x}{l}+C_{1} \sinh \xi_{1} \frac{x}{l}+D_{1} \xi_{1} \frac{x}{l} \sinh \xi_{1} \frac{x}{l}+M_{p}(x)$,

$M(x)=A_{1} \cosh \xi_{1} \frac{x}{l} \times \cos \xi_{2} \frac{x}{l}+B_{1} \cosh \xi_{1} \frac{x}{l} \times \sin \xi_{2} \frac{x}{l}+$

$$
C_{1} \sinh \xi_{1} \frac{x}{l} \times \cos \xi_{2} \frac{x}{l}+D_{1} \sinh \xi_{1} \frac{x}{l} \times \sin \xi_{2} \frac{x}{l}+M_{p}(x),
$$


for $\mathrm{k}_{\mathrm{w}}-4 / \alpha^{2}>0, \mathrm{k}_{\mathrm{W}}-4 / \alpha^{2}=0$, and $\mathrm{k}_{\mathrm{w}}-4 / \alpha^{2}<0$, respectively. Combining $\mathrm{M}(\mathrm{x})$ with Equation (35) yields the following equations:

$$
K_{w} w(x)=\frac{d^{2} M(x)}{d x^{2}}+q(x) \quad(37) \quad K_{w} \frac{d w(x)}{d x}=\frac{d^{3} M(x)}{d x^{3}}+\frac{d q(x)}{d x}
$$

The expression of $\mathrm{M}(\mathrm{x})$ being known, applying Equation (1) yields the shear force, and the combination of $\mathrm{M}(\mathrm{x})$ with Equations (12) and (38) yields the rotations of the cross section.

\subsection{Second-order analysis of uniform Timoshenko beams}

The analysis conducted in this section holds for compressive forces smaller (absolute values) than the buckling load.

\subsubsection{Governing equations}

A uniform beam is analyzed, an elastic Winkler foundation not being considered. The axial force $\mathrm{N}$ (positive in tension) is assumed to be constant in segments of the beam. The equation of static equilibrium is as follows:

$$
\frac{d^{2} M(x)}{d x^{2}}+N \frac{d^{2} w(x)}{d x^{2}}=-q(x)
$$

Combining Equation (39) with the MSFCR (Equation (9)) yields the following:

$$
\left(1+\frac{N}{\kappa G A}\right) \frac{d^{2} M(x)}{d x^{2}}-\frac{N}{E I} M(x)=-q(x)
$$

The solution of Equation (40) yields $M(x)$, which contains the integration constants $C_{1}$ and $C_{2}$. The combination of $M(x)$ with Equation (39) yields following equations:

$$
\begin{aligned}
& N \frac{d w(x)}{d x}=-\frac{d M(x)}{d x}-\int q(x) d x+C_{3} \\
& N w(x)=-M(x)-\iint q(x) d x d x+C_{3} x+C_{4}
\end{aligned}
$$

The transverse force $\mathrm{T}(\mathrm{x})$ is determined as follows:

$$
T(x)=V(x)+N \frac{d w(x)}{d x}
$$

Substituting Equations (1) and (41) into Equation (43) yields the transverse force $\mathrm{T}(\mathrm{x})$ as follows:

$$
T(x)=-\int q(x) d x+C_{3}
$$


The combination of $\mathrm{M}(\mathrm{x})$, Equations (12) and (41) yields the rotation of the cross section as follows:

$$
N \varphi(x)=-\left(1+\frac{N}{\kappa G A}\right) \frac{d M(x)}{d x}-\int q(x) d x+C_{3}
$$

\subsubsection{Element stiffness matrix}

The sign conventions for bending moments, transverse forces, displacements, and rotations adopted for use in determining the element stiffness matrix in local coordinates is illustrated in Figure 4.

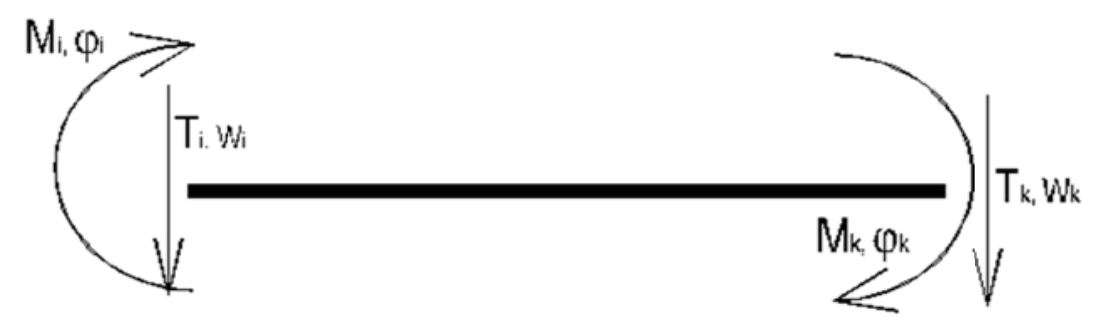

Fig 4 Sign convention for bending moments, transverse forces, displacements, and rotation angles for second-order $4 \times 4$ element stiffness matrix

Let us define following vectors,

$$
\vec{S}=\left[T_{i} ; M_{i} ; T_{k} ; M_{k}\right]^{T}
$$

$$
\vec{V}=\left[w_{i} ; \varphi_{i} ; w_{k} ; \varphi_{k}\right]^{T}
$$

we set

$$
N=k \frac{E I}{l^{2}}
$$

The combination of Equation (40), the bending shear factor (Equation (13)), Equation (47), and the absence of a distributed load yields the following:

$$
(1+k \alpha) \frac{d^{2} M(x)}{d x^{2}}-\frac{k}{l^{2}} M(x)=0
$$

\section{Case 1: Compressive force with $k \alpha>-1$}

The solution of Equation (48) is as follows, with the parameter $\xi 1$ defined as shown:

$$
M(x)=A_{1} \cos \xi_{1} \frac{x}{l}+B_{1} \sin \xi_{1} \frac{x}{l} \quad(49) \quad \xi_{1}=\sqrt{\frac{-k}{1+k \alpha}}
$$


Combining Equations (39) and (49) yields the following:

$$
\begin{aligned}
& N l \frac{d w(x)}{d x}=A_{1} \xi_{1} \sin \xi_{1} \frac{x}{l}-B_{1} \xi_{1} \cos \xi_{1} \frac{x}{l}+N l C_{1} \\
& N w(x)=-A_{1} \cos \xi_{1} \frac{x}{l}-B_{1} \sin \xi_{1} \frac{x}{l}+N C_{1} x+N D_{1}
\end{aligned}
$$

The combination of Equation (12) for the rotation of the cross section with Equations (49) and (51) yields the following, with the parameter $\xi 2$ defined as shown:

$$
\operatorname{Nl} \varphi(x)=A_{1} \xi_{2} \sin \xi_{1} \frac{x}{l}-B_{1} \xi_{2} \cos \xi_{1} \frac{x}{l}+N l C_{1} \quad \text { (53) } \quad \xi_{2}=\sqrt{-k(1+k \alpha)}
$$

By applying Equations (1), (43), (49), and (51), the transverse force $\mathrm{T}(\mathrm{x})$ yields:

$$
T(x)=N C_{1}
$$

Considering the static compatibility boundary conditions (Equations (21) to (34)), whereby the shear forces are replaced by the transverse forces, the geometric compatibility boundary conditions, and Equations (49) to (55), the following equations are obtained:

$$
\begin{aligned}
& T_{i}=-T(x=0) \rightarrow T_{i}=-N C_{1} \\
& M_{i}=M(x=0)=A_{1} \\
& T_{k}=T(x=l) \rightarrow T_{k}=N C_{1} \\
& M_{k}=-M(x=l)=-A_{1} \cos \xi_{1}-B_{1} \sin \xi_{1} \\
& w(x=0)=w_{i} \rightarrow N \times w_{i}=-A_{1}+N D_{1} \\
& \varphi(x=0)=\varphi_{i} \rightarrow N \times \varphi_{i}=-B_{1} \xi_{2} / l+N C_{1} \\
& w(x=l)=w_{k} \rightarrow N \times w_{k}=-A_{1} \cos \xi_{1}-B_{1} \sin \xi_{1}+N l C_{1}+N D_{1} \\
& \varphi(x=l)=\varphi_{k} \rightarrow N \times \varphi_{k}=A_{1} \xi_{2} / l \times \sin \xi_{1}-B_{1} \xi_{2} / l \times \cos \xi_{1}+N C_{1}
\end{aligned}
$$

The combination of Equations (18), (45), (46), and (56) yields the element stiffness matrix as follows:

$$
K_{T b l}=E I \frac{k}{l^{2}} \times\left[\begin{array}{cccc}
0 & 0 & -1 & 0 \\
1 & 0 & 0 & 0 \\
0 & 0 & 1 & 0 \\
-\cos \xi_{1} & -\sin \xi_{1} & 0 & 0
\end{array}\right] \times\left[\begin{array}{cccc}
-1 & 0 & 0 & 1 \\
0 & -\frac{\xi_{2}}{l} & 1 & 0 \\
-\cos \xi_{1} & -\sin \xi_{1} & l & 1 \\
\frac{\xi_{2}}{l} \sin \xi_{1} & -\frac{\xi_{2}}{l} \cos \xi_{1} & 1 & 0
\end{array}\right]^{-1}
$$


The development of Equation (57) yields the following formulation:

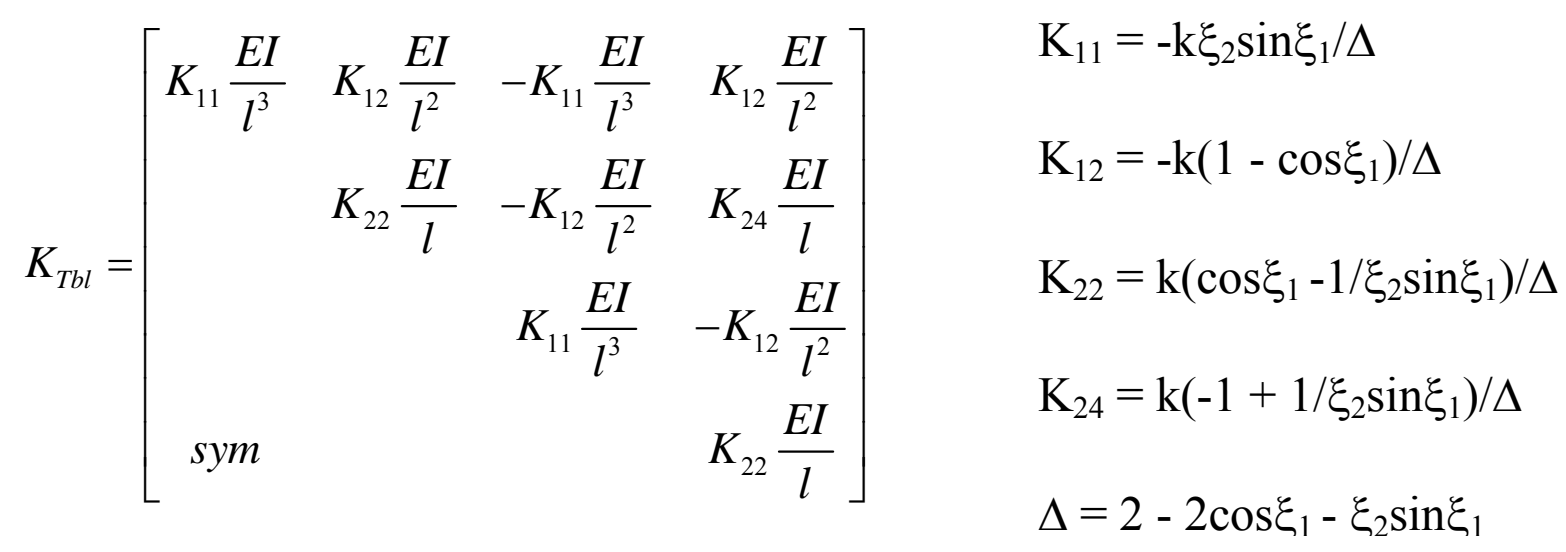

Accordingly, the element stiffness matrix of the beam having a hinge is as follows:

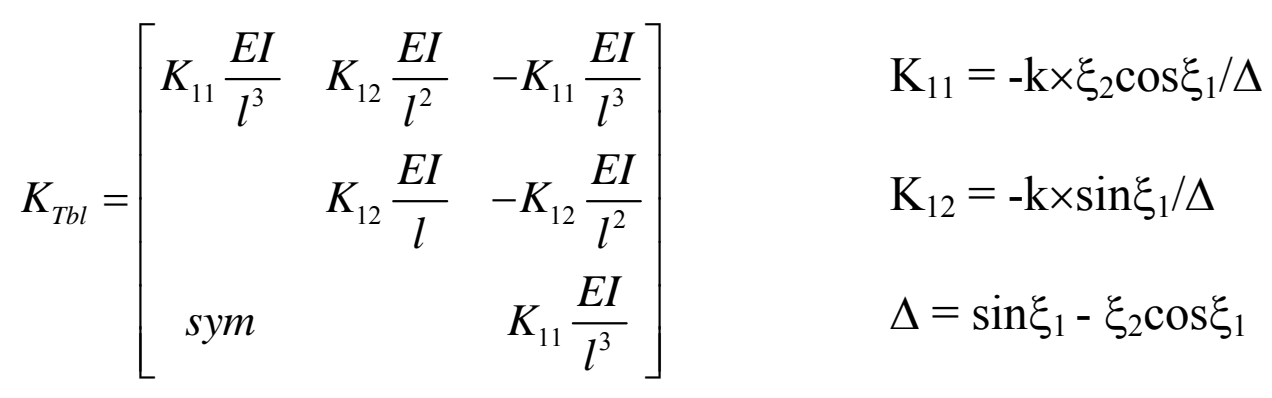

\section{Case 2: Tensile force or compressive force with $k \alpha<-1$}

The solution of Equation (48) is as follows, with the parameter $\xi 3$ defined as shown:

$$
M(x)=A_{2} \cosh \xi_{3} \frac{x}{l}+B_{2} \sinh \xi_{3} \frac{x}{l} \quad(60) \quad \xi_{3}=\sqrt{\frac{k}{1+k \alpha}}
$$

Combining Equations (39) and (60) and integrating with respect to x yields the following:

$$
\begin{aligned}
& N l \frac{d w(x)}{d x}=-A_{2} \xi_{3} \sinh \xi_{3} \frac{x}{l}-B_{2} \xi_{3} \cosh \xi_{3} \frac{x}{l}+N l C_{2} \\
& N w(x)=-A_{2} \cosh \xi_{3} \frac{x}{l}-B_{2} \sinh \xi_{3} \frac{x}{l}+N C_{2} x+N D_{2}
\end{aligned}
$$

Combination of Equation (12) for the rotation of the cross section, with Equations (60) and (62) yields the following:

$N l \varphi(x)=-A_{2} \xi_{4} \sinh \xi_{3} \frac{x}{l}-B_{2} \xi_{4} \cosh \xi_{3} \frac{x}{l}+N l C_{2} \quad$ (64) $\quad \xi_{4}= \pm \sqrt{k(1+k \alpha)}$

The parameter $\xi_{4}$ (Equation (65)) has a positive value in the case of tension and a negative value in the case of compression with $\mathrm{k} \alpha<-1$. Applying Equations (1), (43), (60), and (62), the transverse force $\mathrm{T}(\mathrm{x})$ yields the following:

$$
T(x)=N C_{2}
$$


Considering the static compatibility boundary conditions (Equations (21) to (24)), whereby the shear forces are replaced by the transverse forces, and the geometric compatibility boundary conditions, and Equations (60) to (66), the element stiffness matrix can be expressed as follows:

$$
K_{\text {Tbl }}=E I \frac{k}{l^{2}} \times\left[\begin{array}{cccc}
0 & 0 & -1 & 0 \\
1 & 0 & 0 & 0 \\
0 & 0 & 1 & 0 \\
-\cosh \xi_{1} & -\sinh \xi_{1} & 0 & 0
\end{array}\right] \times\left[\begin{array}{cccc}
-1 & 0 & 0 & 1 \\
0 & -\frac{\xi_{4}}{l} & 1 & 0 \\
-\cosh \xi_{1} & -\sinh \xi_{1} & l & 1 \\
-\frac{\xi_{4}}{l} \sinh \xi_{1} & -\frac{\xi_{4}}{l} \cosh \xi_{1} & 1 & 0
\end{array}\right]^{-1}
$$

The development of Equation (67) yields the following formulation:

$$
K_{\text {Tbl }}=\left[\begin{array}{ccccc}
K_{11} \frac{E I}{l^{3}} & K_{12} \frac{E I}{l^{2}} & -K_{11} \frac{E I}{l^{3}} & K_{12} \frac{E I}{l^{2}} \\
& K_{22} \frac{E I}{l} & -K_{12} \frac{E I}{l^{2}} & K_{24} \frac{E I}{l} \\
& & K_{11} \frac{E I}{l^{3}} & -K_{12} \frac{E I}{l^{2}} \\
& & & K_{22} \frac{E I}{l}
\end{array}\right] \quad \begin{aligned}
& \mathrm{K}_{11}=\mathrm{k} \xi_{4} \sinh \xi_{3} / \Delta \\
& \mathrm{K}_{12}=-\mathrm{k}\left(1-\cosh \xi_{3}\right) / \Delta \\
& \mathrm{K}_{22}=\mathrm{k}\left(\cosh \xi_{3}-1 / \xi_{4} \sinh \xi_{3}\right) / \Delta \\
& \mathrm{K}_{24}=\mathrm{k}\left(-1+1 / \xi_{4} \sinh \xi_{3}\right) / \Delta \\
& \Delta=2-2 \cosh \xi_{3}+\xi_{4} \sinh \xi_{3}
\end{aligned}
$$

Accordingly, the element stiffness matrix of the beam having a hinge is as follows:

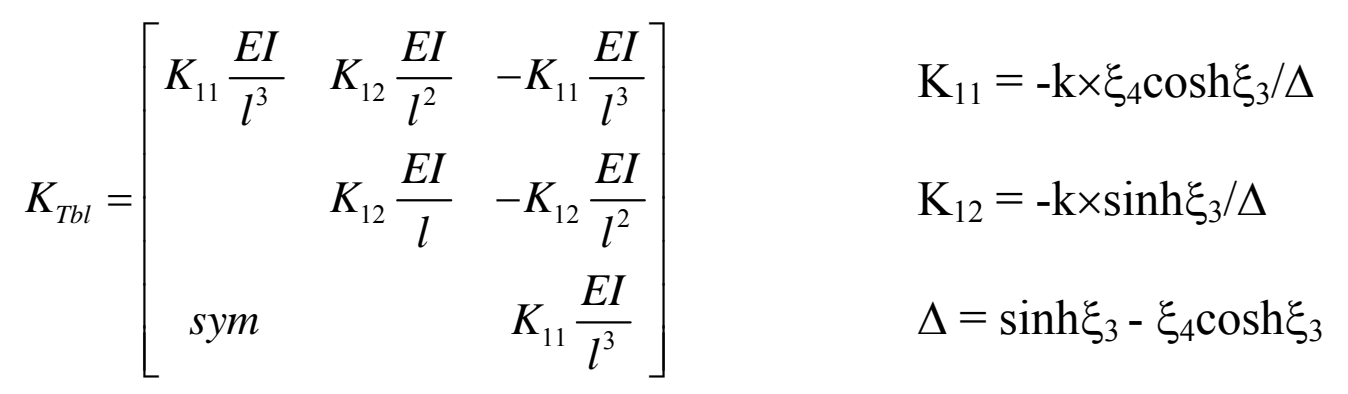

\subsubsection{Analysis of some load cases}

Detailed results for various support conditions and loadings are presented in Appendices E and F.

Uniformly distributed load p. Equation (48) is modified as follows:

$$
(1+k \alpha) \frac{d^{2} M(x)}{d x^{2}}-\frac{k}{l^{2}} M(x)=-p
$$


Case 1: Compressive force with $\mathbf{k} \boldsymbol{\alpha}>\mathbf{- 1}$. The analysis, conducted similarly to Section 2.3.2, yields

$$
\begin{aligned}
& M(x)=A_{1} \cos \xi_{1} \frac{x}{l}+B_{1} \sin \xi_{1} \frac{x}{l}+\frac{p l^{2}}{k}, \quad T(x)=-p x+N C_{1} \\
& N w(x)=-A_{1} \cos \xi_{1} \frac{x}{l}-B_{1} \sin \xi_{1} \frac{x}{l}-\frac{p}{2} x^{2}+N C_{1} x+N D_{1} \\
& N l \varphi(x)=A_{1} \xi_{2} \sin \xi_{1} \frac{x}{l}-B_{1} \xi_{2} \cos \xi_{1} \frac{x}{l}-p l x+N l C_{1}
\end{aligned}
$$

Case 2: Tensile force or compressive force with $\mathbf{k} \alpha<-1$. The analysis yields

$$
\begin{aligned}
& M(x)=A_{2} \cosh \xi_{3} \frac{x}{l}+B_{2} \sinh \xi_{3} \frac{x}{l}+\frac{p l^{2}}{k}, \quad T(x)=-p x+N C_{2} \\
& N w(x)=-A_{2} \cosh \xi_{3} \frac{x}{l}-B_{2} \sinh \xi_{3} \frac{x}{l}-\frac{p}{2} x^{2}+N C_{2} x+N D_{2} \\
& N l \varphi(x)=-A_{2} \xi_{4} \sinh \xi_{3} \frac{x}{l}-B_{2} \xi_{4} \cosh \xi_{3} \frac{x}{l}-p l x+N l C_{2}
\end{aligned}
$$

\section{Non-uniform heating}

Combining Equation (11) and Equation (39) with $\mathrm{q}(\mathrm{x})=0$, yields the modification of Equation (48) as follows:

$$
(1+k \alpha) \frac{d^{2} M(x)}{d x^{2}}-\frac{k}{l^{2}} M(x)=\frac{k}{l^{2}} E I \kappa_{T}
$$

Case 1: Compressive force with $\mathbf{k} \boldsymbol{\alpha}>\mathbf{- 1}$. The analysis, conducted similarly to Section 2.3.2, yields

$$
\begin{aligned}
& M(x)=A_{1} \cos \xi_{1} \frac{x}{l}+B_{1} \sin \xi_{1} \frac{x}{l}-E I \kappa_{T}, \quad T(x)=N C_{1} \\
& N w(x)=-A_{1} \cos \xi_{1} \frac{x}{l}-B_{1} \sin \xi_{1} \frac{x}{l}+N C_{1} x+N D_{1} \\
& N l \varphi(x)=A_{1} \xi_{2} \sin \xi_{1} \frac{x}{l}-B_{1} \xi_{2} \cos \xi_{1} \frac{x}{l}+N l C_{1}
\end{aligned}
$$

Case 2: Tensile force or compressive force with $k \alpha<-1$. The analysis yields

$$
\begin{aligned}
& M(x)=A_{2} \cosh \xi_{3} \frac{x}{l}+B_{2} \sinh \xi_{3} \frac{x}{l}-E I \kappa_{T}, \quad T(x)=N C_{2} \\
& N w(x)=-A_{2} \cosh \xi_{3} \frac{x}{l}-B_{2} \sinh \xi_{3} \frac{x}{l}+N C_{2} x+N D_{2} \\
& N l \varphi(x)=-A_{2} \xi_{4} \sinh \xi_{3} \frac{x}{l}-B_{2} \xi_{4} \cosh \xi_{3} \frac{x}{l}+N l C_{2}
\end{aligned}
$$




\subsubsection{Beam resting on an elastic Winkler foundation with an axial load}

In the equation of static equilibrium, the axial force $\mathrm{N}$ (the value of which is positive in tension) is assumed to be constant in segments of the beam. The stiffness of the Winkler foundation is denoted by $\mathrm{K}_{\mathrm{w}}$.

$$
\frac{d^{2} M(x)}{d x^{2}}+N \frac{d^{2} w(x)}{d x^{2}}-K_{w} w(x)=-q(x)
$$

The combination of Equation (72) with the MSFCR (Equation (9)) yields the following:

$$
K_{w} w(x)=\left(1+\frac{N}{\kappa G A}\right) \frac{d^{2} M(x)}{d x^{2}}-\frac{N}{E I} M(x)+q(x)
$$

Differentiating twice both sides of Equation (73) with respect to $x$ and combining the result with the MSFCR (Equation (9)) yields the following:

$$
\left(1+\frac{N}{\kappa G A}\right) \frac{d^{4} M(x)}{d x^{4}}-\left(\frac{N}{E I}+\frac{K_{w}}{\kappa G A}\right) \frac{d^{2} M(x)}{d x^{2}}+\frac{K_{w}}{E I} M(x)=-\frac{d^{2} q(x)}{d x^{2}}
$$

The solution of Equation (74) yields the formulation of $M(x)$ with four integration constants. From $M(x)$, combined with Equations (1) and (73), the shear force $\mathrm{V}(\mathrm{x})$ and the deflection $\mathrm{w}(\mathrm{x})$ can be deduced. The application of Equations (12) and (43) yields the transverse forces $\mathrm{T}(\mathrm{x})$ and the rotations of the cross section $\varphi(\mathrm{x})$.

\subsubsection{Stability of the Timoshenko beam}

The formulations of bending moment $\mathrm{M}(\mathrm{x})$, deflection $\mathrm{w}(\mathrm{x})$, rotation of the cross section $\varphi(\mathrm{x})$, and transverse force $\mathrm{T}(\mathrm{x})$ are used to determine the buckling load.

For k $\alpha>-1$, Equations (49) to (55) are considered to satisfy the boundary conditions and continuity conditions.

For $k \alpha<-1$, Equations (60) to (66) are considered to satisfy the boundary conditions and continuity conditions.

The resulting eigenvalue problem is solved to determine the buckling loads. 


\section{Results and Discussion}

\subsection{First-order analysis of Timoshenko beams}

\subsubsection{Uniform Timoshenko beam subjected to uniformly distributed load}

Let us calculate the responses of a pinned-pinned beam subjected to a uniformly distributed load as shown in Figure 5.

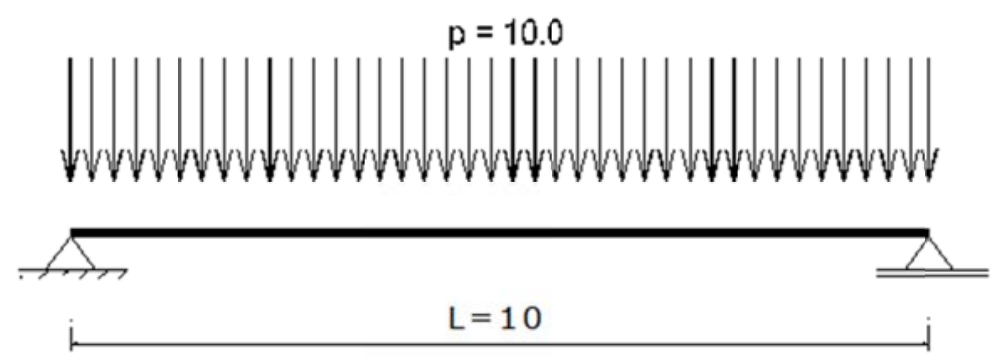

Fig 5 Beam subjected to a uniformly distributed load

The characteristics are as follows: $p=10 \mathrm{kN} / \mathrm{m}, \mathrm{L}=10 \mathrm{~m}, \mathrm{~b}=0.3 \mathrm{~m}, \mathrm{H}=0.5 \mathrm{~m}, \mathrm{E}=34.5 \times 106 \mathrm{kN} / \mathrm{m}^{2}, v=0.3$, and $\kappa=$ 5/6. The calculation of the deflections with the principle of virtual work is presented in Appendix A. Details of the results are presented in the Supplementary Material "Deflection calculation of a pinned-pinned beam using the principle of virtual work." Table 2 lists the results obtained with the principle of virtual work and those obtained in this study. Moreover, closed-form expressions of single-span systems for various support conditions and loadings are presented in Appendix B.

Table 2. Deflections of the beam: Principle of virtual work, and present study

\begin{tabular}{ccc}
\hline $\begin{array}{c}\text { Node } \\
\text { position }\end{array}$ & $\begin{array}{c}\text { Principle of virtual } \\
\text { work (exact results) }\end{array}$ & Present study \\
\hline 0.0 & 0.00000 & 0.00000 \\
1.0 & 0.00382 & 0.00382 \\
2.0 & 0.00722 & 0.00722 \\
3.0 & 0.00988 & 0.00988 \\
4.0 & 0.01157 & 0.01157 \\
5.0 & 0.01215 & 0.01215 \\
6.0 & 0.01157 & 0.01157 \\
7.0 & 0.00988 & 0.00988 \\
8.0 & 0.00722 & 0.00722 \\
9.0 & 0.00382 & 0.00382 \\
10.0 & 0.00000 & 0.00000 \\
\hline
\end{tabular}

The results of this study are exact. 


\section{TIMOSHENKO BEAM THEORY EXACT SOLUTION}

\subsubsection{Beams subjected to concentrated load}

Let us calculate the responses of a uniform fixed-pinned beam subjected to a concentrated load, as shown in Figure 6.

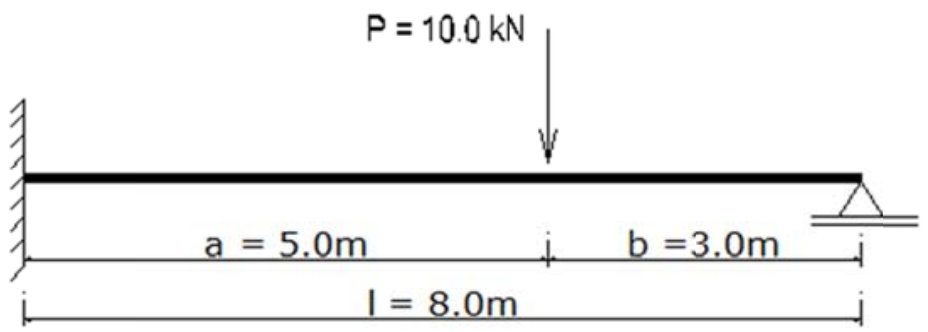

Fig 6 Beam subjected to a concentrated load

Details of the analysis and results are listed in Appendix C and in the Supplementary Material "Analysis of a fixed-pinned beam under concentrated load." Table 3 displays the moments at the fixed end $\left(\mathrm{M}_{\mathrm{FEM}}\right)$ and under the load $\left(\mathrm{M}_{\mathrm{uL}}\right)$ for different values of the bending shear factor, calculated as described in this paper and according to the principle of virtual work (exact values).

Table 3. Moments in beam subjected to a concentrated load

\begin{tabular}{cccccccc}
\hline$\alpha=\mathrm{EI} / \mathrm{KGAl}^{2}=$ & 0.0000 & 0.0250 & 0.0500 & 0.0750 & 0.1000 & 0.1250 & 0.1500 \\
\hline \multicolumn{7}{c}{ Calculations as described in this paper } \\
\hline $\mathrm{M}_{\mathrm{FEM}}=$ & -12.89 & -11.99 & -11.21 & -10.52 & -9.92 & -9.38 & -8.89 \\
$\mathrm{M}_{\mathrm{uL}}=$ & 13.92 & 14.25 & 14.55 & 14.80 & 15.03 & 15.23 & 15.42 \\
\hline \multicolumn{7}{c}{ Calculations according to the principle of virtual work (exact values) } \\
\hline $\mathrm{M}_{\mathrm{FEM}}=$ & -12.89 & -11.99 & -11.21 & -10.52 & -9.92 & -9.38 & -8.89 \\
$\mathrm{M}_{\mathrm{uL}}=$ & 13.92 & 14.25 & 14.55 & 14.80 & 15.03 & 15.23 & 15.42 \\
\hline
\end{tabular}

The results of this study are exact.

\subsection{Second-order analysis of Timoshenko beams}

\subsubsection{Stability of beams}

We determined the buckling loads of uniform single-span beams with various support conditions for different values of the bending shear factor. Details of the analysis and results are listed in Appendix D and in the Supplementary Materials "Buckling analysis of a pinned-pinned beam," "Buckling analysis of a fixed-pinned beam," "Buckling analysis of a fixed-free beam,"and "Buckling analysis of a fixed-fixed beam." 
The buckling load $\mathrm{N}_{\mathrm{cr}}$ is defined as follows:

$$
N_{c r}=-\pi^{2} E I /(\beta l)^{2}
$$

Values of the buckling factor $\beta$ are listed in Table 4. Closed-form expressions of the matrices expressing the boundary conditions are presented in Appendix D. To determine the buckling loads the determinants of those matrices are set to zero. Closed-form expressions of the buckling factors for a pinned-pinned beam, a fixed-free beam, and a fixed-fixed beam are also presented in Appendix D.

Table 4. Buckling factors for Timoshenko beam with various support conditions

\begin{tabular}{|c|c|c|c|c|c|c|c|}
\hline$\alpha=\mathrm{EI} / \kappa \mathrm{KAAl} l^{2}=$ & 0.000 & 0.025 & 0.050 & 0.075 & 0.100 & 0.1250 & 0.150 \\
\hline \multicolumn{8}{|c|}{ Pinned-pinned beam (SS-SS) } \\
\hline$\beta=$ & 1.0000 & 1.1163 & 1.2220 & 1.3192 & 1.4096 & 1.4946 & 1.5750 \\
\hline \multicolumn{8}{|c|}{ Fixed-pinned beam (F-SS) } \\
\hline$\beta=$ & 0.6992 & 0.8716 & 1.0146 & 1.1392 & 1.2510 & 1.3530 & 1.4474 \\
\hline \multicolumn{8}{|c|}{ Fixed-free beam (F-FR) } \\
\hline$\beta=$ & 2.0000 & 2.0608 & 2.1198 & 2.1772 & 2.2332 & 2.2877 & 2.3410 \\
\hline \multicolumn{8}{|c|}{ Fixed-fixed beam (F-F) } \\
\hline$\beta=$ & 0.5000 & 0.7048 & 0.8623 & 0.9951 & 1.1122 & 1.2181 & 1.3155 \\
\hline
\end{tabular}

We recall that the exact values of the buckling factors $\beta$ for $\alpha=0.0$ (corresponding to the Euler-Bernoulli beam) for the support conditions SS-SS, F-SS, F-FR and F-F are 1.00, 0.700, 2.00, and 0.500, respectively.

\subsubsection{Beams subjected to uniformly distributed load and an axial force}

Let us calculate the responses of a uniform pinned-pinned beam subjected to a uniformly distributed load and an axial force, as shown in Figure 7. 


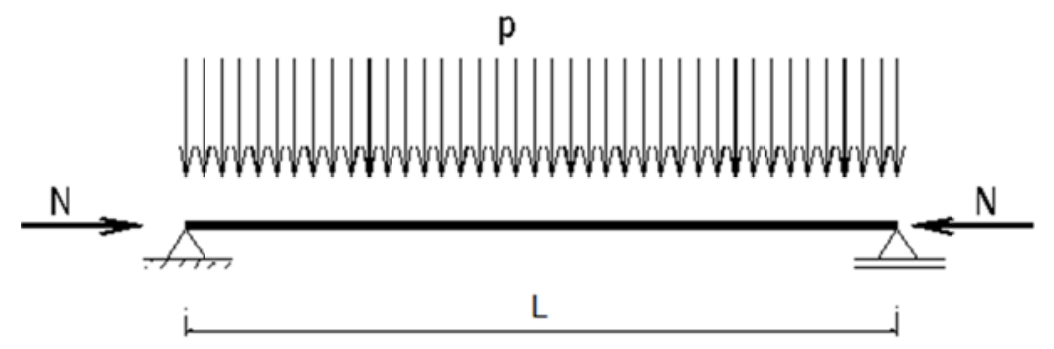

Fig 7 Beam subjected to a uniformly distributed load and an axial force

The bending moments at mid-span (position $\mathrm{L} / 2$ ) are calculated for different values of axial force (coefficient $\mathrm{k}$ ) and bending shear factor $\alpha$, and the results are listed in Table 5. Closed-form expressions of bending moment and deflection curves are presented in Appendix E, together with those related to other support conditions and loadings. Details of the results are presented in the Supplementary Material "Bending analysis of a pinned-pinned beam subjected to a uniformly distributed load and an axial force."

Table 5. Moments at position $\mathrm{L} / 2$ for different values of shear factor and axial load

\begin{tabular}{cccccccc}
\hline \multicolumn{2}{c}{$\alpha=0.025$} & \multicolumn{2}{c}{$\alpha=0.05$} & \multicolumn{2}{c}{$\alpha=0.075$} & \multicolumn{2}{c}{$\alpha=0.10$} \\
$\mathrm{k}=$ & $\mathrm{MMP} / \mathrm{pl}^{2}=$ & $\mathrm{k}=$ & $\mathrm{MMP} / \mathrm{pl}^{2}=$ & $\mathrm{k}=$ & $\mathrm{MMP} / \mathrm{pl}^{2}=$ & $\mathrm{k}=$ & $\mathrm{MMP} / \mathrm{pl}^{2}=$ \\
\hline-7.50 & 2.4474 & -6.50 & 7.8536 & & & \\
-6.00 & 0.5278 & -6.00 & 1.3947 & -5.50 & 4.2593 & & \\
-5.00 & 0.3453 & -5.00 & 0.5242 & -5.00 & 1.0825 & -4.50 & 1.3635 \\
-4.00 & 0.2561 & -4.00 & 0.3215 & -4.00 & 0.4316 & -4.00 & 0.6553 \\
-3.00 & 0.2032 & -3.00 & 0.2313 & -3.00 & 0.2684 & -3.00 & 0.3197 \\
-2.00 & 0.1683 & -2.00 & 0.1804 & -2.00 & 0.1944 & -2.00 & 0.2108 \\
-1.00 & 0.1435 & -1.00 & 0.1477 & -1.00 & 0.1522 & -1.00 & 0.1570 \\
0.00 & 0.1250 & 0.00 & 0.1250 & 0.00 & 0.1250 & 0.00 & 0.1250 \\
1.00 & 0.1107 & 1.00 & 0.1083 & 1.00 & 0.1060 & 1.00 & 0.1038 \\
2.00 & 0.0993 & 2.00 & 0.0955 & 2.00 & 0.0920 & 2.00 & 0.0887 \\
3.00 & 0.0900 & 3.00 & 0.0854 & 3.00 & 0.0812 & 3.00 & 0.0774 \\
4.00 & 0.0822 & 4.00 & 0.0772 & 4.00 & 0.0727 & & \\
\hline
\end{tabular}

The limits of $\mathrm{k}$ corresponding to buckling are listed in the Supplementary Material "Buckling analysis of a pinned-pinned beam". 


\section{TIMOSHENKO BEAM THEORY EXACT SOLUTION}

\subsection{2a Beams subjected to concentrated load}

Let us calculate the responses of a uniform fixed-pinned beam subjected to a concentrated load and an axial load, as shown in Figure 8. The compressive forces are assumed smaller (absolute values) than the buckling load.

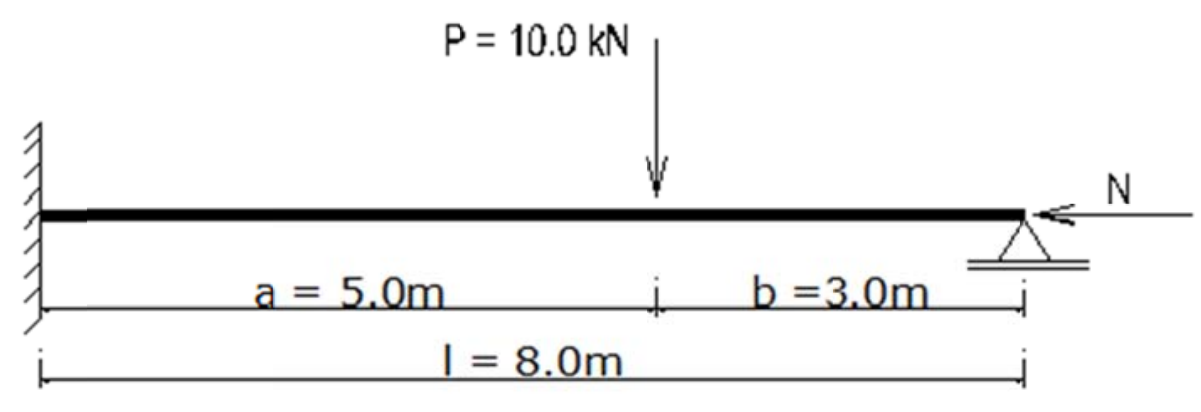

Fig 8 Beam subjected to a concentrated load and an axial force

Details of the analysis and results are listed in Appendix F and in the Supplementary Material "Bending analysis of a fixed-pinned beam subjected to a concentrated load and an axial force." Furthermore, closed-form expressions of bending moments and deflections for other support conditions are presented in Appendix F. Table 6 lists the fixed-end moments $\left(\mathrm{M}_{\mathrm{FEM}}\right)$ and under the load $\left(\mathrm{M}_{\mathrm{uL}}\right)$ for different values of bending shear factor and axial force.

Table 6. Moments in beam subjected to a concentrated load and an axial force

\begin{tabular}{ccccccc}
\hline & \multicolumn{3}{l}{ Coefficient of axial force $\mathrm{k}=-4.0$} & \multicolumn{3}{l}{ Coefficient of axial force $\mathrm{k}=-6.0$} \\
\hline$\alpha=\mathrm{EI} / \mathrm{\kappa GAl}^{2}=$ & 0.0000 & 0.0250 & 0.0500 & 0.0000 & 0.0250 & 0.0500 \\
$\mathrm{M}_{\mathrm{FEM}}=$ & -15.65 & -16.99 & -18.98 & -17.60 & -21.58 & -29.28 \\
$\mathrm{M}_{\mathrm{uL}}=$ & 16.73 & 19.72 & 23.70 & 18.72 & 24.68 & 35.46 \\
\hline \multicolumn{3}{l}{ Coefficient of axial force } & $\mathrm{k}=4.0$ & Coefficient of axial force $\mathrm{k}=6.0$ \\
\hline$\alpha=\mathrm{EI} / \mathrm{\kappa GAl}^{2}=$ & 0.0000 & 0.0250 & 0.0500 & 0.0000 & 0.0250 & 0.0500 \\
$\mathrm{M}_{\mathrm{FEM}}=$ & -11.04 & -9.31 & -7.98 & -10.32 & -8.39 & -6.98 \\
$\mathrm{M}_{\mathrm{uL}}=$ & 12.03 & 11.27 & 10.60 & 11.29 & 10.22 & 9.35 \\
\hline
\end{tabular}

\subsubsection{Second-order element stiffness matrix}

Let us calculate the second-order element stiffness matrix of a beam with the following characteristics:

$\mathrm{k}=-1.5$ (Equation (47)), $\alpha=0.05$ (Equation (13)), and length $\mathrm{L}=4.0 \mathrm{~m}$. The matrix is calculated using Equations (57) and (58). Details of the results are presented in the Supplementary Material "Second-order element stiffness matrix." The calculation of the element stiffness matrix of this Timoshenko beam yields: 


$$
K_{T b l}=E I \times\left[\begin{array}{cccc}
0.0917 & 0.2303 & -0.0917 & 0.2303 \\
0.2303 & 0.6759 & -0.2303 & 0.2454 \\
-0.0917 & -0.2303 & 0.0917 & -0.2303 \\
0.2303 & 0.2454 & -0.2303 & 0.6759
\end{array}\right]
$$

Let us now calculate the element stiffness matrix of the beam with the formula presented by Hu et al. (2019):

$$
K_{\text {Ibl }}=E I \chi \chi\left[\begin{array}{cccc}
0 & 0 & (\lambda / L)^{2} & 0 \\
(\lambda / L)^{2} & 0 & 0 & 0 \\
0 & 0 & -(\lambda / L)^{2} & 0 \\
-(\lambda / L)^{2} \cos \lambda & -(\lambda / L)^{2} \sin \lambda & 0 & 0
\end{array}\right] \times\left[\begin{array}{cccc}
1 & 0 & 0 & 1 \\
0 & \chi \lambda / L & 1 & 0 \\
\cos \lambda & \sin \lambda & L & 1 \\
-\chi \lambda / L \sin \lambda & \chi \lambda / L \cos \lambda & 1 & 0
\end{array}\right]^{-1}
$$

The aforementioned characteristics become $\quad \mathrm{P}=1.5 \times \mathrm{EI} / \mathrm{L}^{2}, \quad \chi=1-\mathrm{P} /(\mathrm{ksGA})=1-1.5 \times 0.05=0.925$,

$$
\text { and } \quad \lambda=\sqrt{P L^{2} / \chi E I}=\sqrt{1.5 / 0.925}=1.273
$$

Details of the calculations are presented in the abovementioned Supplementary Material.

$$
K_{T b l}=E I \times\left[\begin{array}{cccc}
0.0917 & 0.2303 & -0.0917 & 0.2303 \\
0.2303 & 0.6759 & -0.2303 & 0.2454 \\
-0.0917 & -0.2303 & 0.0917 & -0.2303 \\
0.2303 & 0.2454 & -0.2303 & 0.6759
\end{array}\right]
$$

The results of this study are in good agreement with those of $\mathrm{Hu}$ et al. (2019).

In fact, both formulas are identical since following equivalences exist between the parameters considered by $\mathrm{Hu}$ et al. (2019) $(\chi, \lambda)$, and those considered in the present study $\left(\mathrm{k}, \alpha, \xi_{1}, \xi_{2}\right): \chi=1+\mathrm{k} \alpha, \lambda=\xi_{1}, \chi \lambda=\xi_{2}, \chi(\lambda / \mathrm{L})^{2}=-\mathrm{k} / \mathrm{L}^{2}$.

The equations for the determination of the buckling loads (see Appendix D) are also identical to those of Hu et al. (2019) (Table 1). However, the formula presented by Hu et al. (2019) only applies for compressive forces with k $\alpha>-1$. For this beam subjected to a tensile force $(\mathrm{k}=1.5$ (Equation (47))), the stiffness matrix becomes (Equation (67):

$$
K_{T b l}=E I \times\left[\begin{array}{cccc}
0.8850 & 1.4784 & -0.8850 & 1.4784 \\
1.4784 & 4.6884 & -1.4784 & 1.2253 \\
-0.8850 & -1.4784 & 0.8850 & -1.4784 \\
1.4784 & 1.2253 & -1.4784 & 4.6884
\end{array}\right]
$$




\section{TIMOSHENKO BEAM THEORY EXACT SOLUTION}

\section{Conclusion}

The moment-shear force-curvature relationship presented in this study enabled the derivation of closed-form solutions for first-order analysis, second-order analysis, and stability of Timoshenko beams. The results showed that the calculations conducted as described in this paper are exact. Closed-form expressions of efforts and deformations for various load cases were presented, as well as closed-form expressions of second-order element stiffness matrices (the axial force being tensile or compressive) in local coordinates. The determination of element stiffness matrices (ESM) enables the analysis of systems with the direct stiffness method. We showed that ESM can also be determined by the presence of hinges.

\section{Calculation of bending moments and shear forces:}

Influence of tensile force: With increasing tensile force, bending moments decrease (in absolute values), and with increasing bending shear factor, bending moments decrease (in absolute values).

Influence of compressive force: With increasing compressive force, bending moments increase (in absolute values), and with increasing bending shear factor, bending moments also increase (in absolute values).

Stability of the beam: With increasing bending shear factor, the buckling load decreases.

The following aspects not addressed in this study could be examined in future research:

Analysis of linear structures, such as frames, through the transformation of element stiffness matrices from local coordinates in global coordinates.

Second-order analysis of frames free to sidesway with consideration of P- $\Delta$ effect.

Use of the direct stiffness method, since element stiffness matrices are presented.

Analysis of positions of discontinuity (interior supports, springs, hinges, abrupt change of section), since closedform expressions of bending moments, shear or transverse forces, rotation angles, and deflection are known.

Beams resting on Pasternak foundations, the Pasternak soil parameter can be considered as a tensile force.

Supplementary Materials: The following files were uploaded during submission:

- "Deflection calculation of a pinned-pinned beam using the principle of virtual work,"

- "Analysis of a fixed-pinned beam under concentrated load,"

- "Buckling analysis of a pinned-pinned beam," 
- "Buckling analysis of a fixed-pinned beam,"

- "Buckling analysis of a fixed-free beam,"

- "Buckling analysis of a fixed-fixed beam,"

- "Bending analysis of a pinned-pinned beam subjected to a uniformly distributed load and an axial force,"

- "Bending analysis of a fixed-pinned beam subjected to a concentrated load and an axial force," and

- "Second-order element stiffness matrix."

\section{Funding:}

\section{Acknowledgments:}

Conflicts of Interest: The author declares no conflict of interest.

\section{Appendix A Deflection calculation at position $\mathrm{x}_{0}$ with the principle of virtual work}

$$
\delta\left(x_{0}\right)=\int \frac{M(x) \times m(x)}{E I} d x+\int \frac{V(x) \times v(x)}{\kappa G A} d x
$$

$\mathrm{M}(\mathrm{x}), \mathrm{V}(\mathrm{x})$ are the bending moment and shear force due to the distributed load, respectively. $\mathrm{m}(\mathrm{x}), \mathrm{v}(\mathrm{x})$ are the bending moment and shear force due to a virtual unit load at the point of interest $\mathrm{x}_{0}$, respectively.

$\mathrm{M}(\mathrm{x})=\mathrm{px}(1-\mathrm{x}) / 2 \quad \mathrm{~V}(\mathrm{x})=\mathrm{p}(1 / 2-\mathrm{x})$

$\mathrm{m}(\mathrm{x})=\mathrm{x}\left(1-\mathrm{x}_{0}\right) / 1$ for $\mathrm{x} \leq \mathrm{x}_{0}$

$$
\mathrm{m}(\mathrm{x})=\mathrm{x}_{0}(1-\mathrm{x}) / 1 \text { for } \mathrm{x} \geq \mathrm{x}_{0}
$$

$\mathrm{v}(\mathrm{x})=1-\mathrm{x}_{0} / 1$ for $\mathrm{x}<\mathrm{x}_{0}$

$$
\mathrm{v}(\mathrm{x})=-\mathrm{x}_{0} / 1 \quad \text { for } \mathrm{x}>\mathrm{x}_{0}
$$

Substituting Equations (A2) into Equation (A1) yields,

$$
\delta\left(x_{0}\right)=\frac{1}{24 E I} p l^{2} x_{0}\left(l-x_{0}\right)\left[1+\frac{x_{0}}{l} \frac{l-x_{0}}{l}\right]+\frac{1}{2 \kappa G A} x_{0}\left(1-\frac{x_{0}}{l}\right) p l
$$




\section{Appendix B Closed-form expressions of single-span systems for various support conditions and loadings}

The equations of Sections 2.2.1 and 2.2.2 are applied, and the corresponding boundary conditions are satisfied. Closedform expressions of bending moment and deflection curves are as follows:

\section{Pinned-pinned beam}

- Uniformly distributed load $\mathbf{p}$

$$
\begin{aligned}
& M(x)=-\frac{p}{2} x^{2}+\frac{p l}{2} x \\
& E I \times w(x)=\frac{p}{24} x^{4}-\frac{p l}{12} x^{3}-\alpha \frac{p l^{2}}{2} x^{2}+\left(\frac{1}{24}+\frac{\alpha}{2}\right) p l^{3} x
\end{aligned}
$$

- Triangular distributed load (zero at $x=0$, and $p$ at $x=1)$

$$
\begin{aligned}
& M(x)=-\frac{p}{6 l} x^{3}+\frac{p l}{6} x \\
& E I \times w(x)=\frac{p}{120 l} x^{5}-\left(\frac{1}{6}+\alpha\right) \frac{p l}{6} x^{3}+\left(\frac{7}{360}+\frac{\alpha}{6}\right) p l^{3} x
\end{aligned}
$$

- Non-uniform heating

$$
M(x)=0 \quad E I \times w(x)=-\frac{E I \kappa_{T}}{2} x^{2}+\frac{E I \kappa_{T}}{2} l x
$$

\section{Fixed-Free beam}

- Uniformly distributed load p

$$
\begin{aligned}
& M(x)=-\frac{p}{2} x^{2}+p l x-\frac{p l^{2}}{2} \\
& E I \times w(x)=\frac{p}{24} x^{4}-\frac{p l}{6} x^{3}-\left(\alpha-\frac{1}{2}\right) \frac{p l^{2}}{2} x^{2}+\alpha p l^{3} x
\end{aligned}
$$

- Triangular distributed load (zero at $x=0$, and $p$ at $x=1)$

$$
\begin{aligned}
& M(x)=-\frac{p}{6 l} x^{3}+\frac{p l}{2} x-\frac{p l^{2}}{3} \\
& E I \times w(x)=\frac{p}{120 l} x^{5}-\left(\frac{1}{2}+\alpha\right) \frac{p l}{6} x^{3}+\frac{p l^{2}}{6} x^{2}+\alpha \frac{p l^{3}}{2} x
\end{aligned}
$$

- Non-uniform heating

$$
M(x)=0 \quad E I \times w(x)=-\frac{E I \kappa_{T}}{2} x^{2}
$$




\section{Fixed-pinned}

- Uniformly distributed load p

$$
\begin{aligned}
& M(x)=-\frac{p}{2} x^{2}+A p l x+B p l^{2} \\
& E I \times w(x)=\frac{p}{24} x^{4}-A \frac{p l}{6} x^{3}-(B+\alpha) \frac{p l^{2}}{2} x^{2}+\alpha A p l^{3} x \\
& A=\frac{5+12 \alpha}{8(1+3 \alpha)} \quad B=\frac{-1}{8(1+3 \alpha)}
\end{aligned}
$$

- Triangular distributed load (zero at $x=0$, and $p$ at $x=1)$

$$
\begin{aligned}
& M(x)=-\frac{p}{6 l} x^{3}+A p l x+B p l^{2} \\
& E I \times w(x)=\frac{p}{120 l} x^{5}-(A+\alpha) \frac{p l}{6} x^{3}-B \frac{p l^{2}}{2} x^{2}+\alpha A p l^{3} x \\
& A=\frac{9+20 \alpha}{40(1+3 \alpha)} \quad B=\frac{-14}{240(1+3 \alpha)}
\end{aligned}
$$

- Non-uniform heating

$$
\begin{array}{ll}
M(x)=A_{1} x+B_{1} & E I \times w(x)=-\frac{A_{1}}{6} x^{3}-\frac{1}{2}\left(B_{1}+E I \kappa_{T}\right) x^{2}+\alpha l^{2} A_{1} x \\
A_{1}=\frac{E I \kappa_{T}}{2 l(1 / 3+\alpha)} & B_{1}=-\frac{E I \kappa_{T}}{2(1 / 3+\alpha)}
\end{array}
$$

\section{Fixed-fixed beam}

- Uniformly distributed load p

$$
\begin{aligned}
& M(x)=-\frac{p}{2} x^{2}+\frac{p l}{2} x-\frac{p l^{2}}{12} \\
& E I \times w(x)=\frac{p}{24} x^{4}-\frac{p l}{12} x^{3}-\left(\alpha-\frac{1}{12}\right) \frac{p l^{2}}{2} x^{2}+\alpha \frac{p l^{3}}{2} x
\end{aligned}
$$

- Triangular distributed load (zero at $x=0$, and $p$ at $x=1)$

$$
\begin{aligned}
& M(x)=-\frac{p}{6 l} x^{3}+A p l x+B p l^{2} \\
& E I \times w(x)=\frac{p}{120 l} x^{5}-(A+\alpha) \frac{p l}{6} x^{3}-B \frac{p l^{2}}{2} x^{2}+\alpha A p l^{3} x \\
& A=\frac{0.15+2 \alpha}{1+12 \alpha} \quad B=-\frac{0.8+12 \alpha}{24(1+12 \alpha)}
\end{aligned}
$$


- Non-uniform heating

$$
M(x)=-E I \kappa_{T} \quad E I \times w(x)=0
$$

\section{Appendix C Fixed-pinned Timoshenko beam subjected to a concentrated load}

Equations (19), (20), and (20a) are applied on both sides of the concentrated load, $x_{1}$ at the left side $\left(x_{1} \leq \mathrm{a}\right)$ and $x_{2}$ at the right side $\left(x_{2} \leq \mathrm{b}\right)$ :

$$
\begin{aligned}
& M\left(x_{1}\right)=A_{1} x_{1}+B_{1} \quad V\left(x_{1}\right)=A_{1} \\
& E I \times w\left(x_{1}\right)=-\frac{A_{1}}{6} x_{1}^{3}-\frac{B_{1}}{2} x_{1}^{2}+C_{1} x_{1}+D_{1} \\
& E I \times \varphi(x)=-\frac{A_{1}}{2} x^{2}-B_{1} x-\alpha l^{2} A_{1}+C_{1}
\end{aligned}
$$

Similar equations are applied on the right side (with the variable $x_{2}$ ). Following boundary conditions and continuity conditions are applied:

$$
\begin{array}{lll}
\checkmark & \mathrm{w}\left(\mathrm{x}_{1}=0\right)=0: & \mathrm{D}_{1}=0 \\
\checkmark & \varphi\left(\mathrm{x}_{1}=0\right)=0: & -\alpha l^{2} \times \mathrm{A}_{1}+\mathrm{C}_{1}=0 \\
\checkmark & \mathrm{w}\left(\mathrm{x}_{1}=\mathrm{a}\right)=\mathrm{w}\left(\mathrm{x}_{2}=0\right): & -\mathrm{A}_{1} / 6 \times \mathrm{a}^{3}-\mathrm{B}_{1} / 2 \times \mathrm{a}^{2}+\mathrm{C}_{1} \times \mathrm{a}+\mathrm{D}_{1}=\mathrm{D}_{2} \\
\checkmark & \varphi\left(\mathrm{x}_{1}=\mathrm{a}\right)=\varphi\left(\mathrm{x}_{2}=0\right): & -\mathrm{A}_{1} / 2 \times \mathrm{a}^{2}-\mathrm{B}_{1} \times \mathrm{a}+\mathrm{C}_{1}-\alpha l^{2} \times \mathrm{A}_{1}=\mathrm{C}_{2}-\alpha l^{2} \times \mathrm{A}_{2} \\
\checkmark & \mathrm{M}\left(\mathrm{x}_{1}=\mathrm{a}\right)=\mathrm{M}\left(\mathrm{x}_{2}=0\right): & \mathrm{A}_{1} \times \mathrm{a}+\mathrm{B}_{1}=\mathrm{B}_{2} \\
\checkmark & \mathrm{Q}\left(\mathrm{x}_{1}=\mathrm{a}\right)-\mathrm{Q}\left(\mathrm{x}_{2}=0\right)=\mathrm{P}: & \mathrm{A}_{1}-\mathrm{A}_{2}=\mathrm{P} \\
\checkmark & \mathrm{M}\left(\mathrm{x}_{2}=\mathrm{b}\right)=0: & \mathrm{A}_{2} \times \mathrm{b}+\mathrm{B}_{2}=0 \\
\checkmark & \mathrm{w}\left(\mathrm{x}_{2}=\mathrm{b}\right)=0: & -\mathrm{A}_{2} / 6 \times \mathrm{b}^{3}-\mathrm{B}_{2} / 2 \times \mathrm{b}^{2}+\mathrm{C}_{2} \times \mathrm{b}+\mathrm{D}_{2}=0
\end{array}
$$

The unknowns are determined by solving the system of equations, and the moments can be calculated as follows:

Fixed-end moment $\mathrm{M}_{\mathrm{FEM}}=\mathrm{B}_{1}$, and moment under the load $\mathrm{M}_{\mathrm{UL}}=\mathrm{B}_{2}$.

For calculation with the principle of virtual work the fixed-end moment and the moment under the load can be expressed as follows:

$$
M_{F E M}=-\frac{(1+b / l) \times a / l \times b / l}{6 \times\left(1 / 3+E I / \kappa G A l^{2}\right)} \times P l \quad M_{u L}=b / l \times M_{F E M}+P a b / l
$$


For other support conditions (pinned-pinned, fixed-free, and fixed-fixed) the boundary conditions (Equations (C2)a, $(\mathrm{C} 2)_{\mathrm{b}},(\mathrm{C} 2)_{\mathrm{g}}$, and $\left.(\mathrm{C} 2)_{\mathrm{h}}\right)$ are modified accordingly, and the bending moment and deflection curves are deduced using Equation (15c) with $\mathrm{q}=0$.

\section{Appendix D Buckling loads of single-span beams with various support conditions}

Compressive force with $k \alpha<-1$ : The eigenvalue problem led to following equations: $\sinh \xi_{3}=0$, $\sinh \xi_{3}-\xi_{4} \cosh \xi_{3}=0, \cosh \xi_{3}=0$, and $2-2 \cosh \xi_{3}+\xi_{4} \sinh \xi_{3}=0$ for a pinned-pinned beam, a fixedpinned beam, a fixed-free beam, and a fixed-fixed beam, respectively. Recalling that $\xi_{4}$ is negative, those equations have no nontrivial solutions; consequently, no buckling occurred due to the action of a compressive force with $k \alpha<-1$.

\section{Compressive force with $k \alpha>-1$}

Pinned-pinned beam: the results are presented in the Supplementary Material "Buckling analysis of a pinned-pinned beam." To determine the buckling load, the determinant of the matrix $M$ expressing the boundary conditions is set to zero. The matrix $M$, the determinant equation, and the buckling factor $\beta$ are as follows:

$$
M=\left[\begin{array}{cccc}
-1.00 & 0.00 & 0.00 & 1.00 \\
1.00 & 0.00 & 0.00 & 0.00 \\
\cos \xi_{1} & \sin \xi_{1} & 0.00 & 0.00 \\
-\cos \xi_{1} & -\sin \xi_{1} & 1.00 & 1.00
\end{array}\right] \quad \sin \xi_{1}=0, \quad \beta=\sqrt{1+\alpha \pi^{2}}
$$

Fixed-pinned beam: the results are presented in the Supplementary Material "Buckling analysis of a fixed-pinned beam," with the matrix and the equation as follow:

$$
M=\left[\begin{array}{cccc}
-1.00 & 0.00 & 0.00 & 1.00 \\
0.00 & -\xi_{2} & 1.00 & 0.00 \\
\cos \xi_{1} & \sin \xi_{1} & 0.00 & 0.00 \\
-\cos \xi_{1} & -\sin \xi_{1} & 1.00 & 1.00
\end{array}\right] \quad \rightarrow \xi_{1} \sin \xi_{1}+k \cos \xi_{1}=0
$$


Fixed-free beam: the results are presented in the Supplementary Material "Buckling analysis of a fixed-free beam," with the matrix, the equation and the buckling factor $\beta$ as follows

$$
M=\left[\begin{array}{cccc}
-1.00 & 0.00 & 0.00 & 1.00 \\
0.00 & -\xi_{2} & 1.00 & 0.00 \\
\cos \xi_{1} & \sin \xi_{1} & 0.00 & 0.00 \\
0.00 & 0.00 & 1.00 & 0.00
\end{array}\right] \quad \cos \xi_{1}=0, \quad \beta=\sqrt{4+\alpha \pi^{2}}
$$

Fixed-fixed beam: the results are presented in the Supplementary Material "Buckling analysis of a fixed-fixed beam," with the matrix and the equations as follows:

$$
\begin{aligned}
& M=\left[\begin{array}{cccc}
-1.00 & 0.00 & 0.00 & 1.00 \\
0.00 & -\xi_{2} & 1.00 & 0.00 \\
-\cos \xi_{1} & -\sin \xi_{1} & 1.00 & 1.00 \\
\xi_{2} \sin \xi_{1} & -\xi_{2} \cos \xi_{1} & 1.00 & 0.00
\end{array}\right] \quad \rightarrow \sin \frac{\xi_{1}}{2} \times\left(2 \sin \frac{\xi_{1}}{2}+\frac{k}{\xi_{1}} \cos \frac{\xi_{1}}{2}\right)=0 \\
& \sin \frac{\xi_{1}}{2}=0 \rightarrow \beta=\frac{1}{2} \sqrt{1+4 \pi^{2} \alpha}, \quad 2 \sin \frac{\xi_{1}}{2}+\frac{k}{\xi_{1}} \cos \frac{\xi_{1}}{2}=0
\end{aligned}
$$

\section{Appendix E Timoshenko beams subjected to various load cases and an axial force}

The compressive forces are assumed smaller (absolute values) than the buckling load.

Case 1: Compressive force with $k \alpha>\mathbf{- 1}$. The bending moment and the deflection curve are denoted by $\mathrm{M}_{\mathrm{cl}}(\mathrm{x})$ and $\mathrm{W}_{\mathrm{cl}}(\mathrm{x})$, respectively. Let us recall the parameters $\xi_{1}$ and $\xi_{2}$ defined in Equations (50) and (54), respectively.

$$
\xi_{1}=\sqrt{-k /(1+k \alpha)} \quad \xi_{2}=\sqrt{-k(1+k \alpha)}
$$

Case 2: Tensile force or compressive force with $k \alpha<-1$. The bending moment and the deflection curve are denoted by $\mathrm{M}_{\mathrm{c} 2}(\mathrm{x})$ and $\mathrm{w}_{\mathrm{c} 2}(\mathrm{x})$, respectively. Let us recall the parameters $\xi_{3}$ and $\xi_{4}$ defined in Equations (61) and (65), respectively.

$$
\xi_{3}=\sqrt{k /(1+k \alpha)} \quad \xi_{4}= \pm \sqrt{k(1+k \alpha)}
$$

The parameter $\xi_{4}$ (Equation (65)) has a positive value in the case of tension and a negative value in the case of compression with $\mathrm{k} \alpha<-1$. 


\section{Pinned-pinned beam}

- Uniformly distributed load

$$
\begin{aligned}
& M_{c 1}(x)=-\frac{p l^{2}}{k} \cos \xi_{1} \frac{x}{l}-\frac{1-\cos \xi_{1}}{\sin \xi_{1}} \frac{p l^{2}}{k} \sin \xi_{1} \frac{x}{l}+\frac{p l^{2}}{k} \\
& E I \times w_{c 1}(x)=\frac{p l^{4}}{k^{2}} \cos \xi_{1} \frac{x}{l}+\frac{p l^{4}}{k^{2}} \frac{1-\cos \xi_{1}}{\sin \xi_{1}} \sin \xi_{1} \frac{x}{l}-\frac{p l^{2}}{2 k} x^{2}+\frac{p l^{3}}{2 k} x-\frac{p l^{4}}{k^{2}} \\
& M_{c 2}(x)=-\frac{p l^{2}}{k} \cosh \xi_{3} \frac{x}{l}-\frac{1-\cosh \xi_{3}}{\sinh \xi_{3}} \frac{p l^{2}}{k} \sinh \xi_{3} \frac{x}{l}+\frac{p l^{2}}{k} \\
& E I \times w_{c 2}(x)=\frac{p l^{4}}{k^{2}} \cosh \xi_{3} \frac{x}{l}+\frac{p l^{4}}{k^{2}} \frac{1-\cosh \xi_{3}}{\sinh \xi_{3}} \sinh \xi_{3} \frac{x}{l}-\frac{p l^{2}}{2 k} x^{2}+\frac{p l^{3}}{2 k} x-\frac{p l^{4}}{k^{2}}
\end{aligned}
$$

For $\mathrm{k} \alpha=-1$, using Equations (40) and (47) yields the bending moment $\mathrm{M}(\mathrm{x})=\mathrm{pl}^{2} / \mathrm{k}=-\alpha \mathrm{pl}^{2}$.

\section{- Triangular distributed load (zero at $x=0$, and $p$ at $x=1)$}

$$
\begin{aligned}
& M_{c 1}(x)=-\frac{p l^{2}}{k \sin \xi_{1}} \sin \xi_{1} \frac{x}{l}+\frac{p l}{k} x \\
& E I \times w_{c 1}(x)=\frac{p l^{4}}{k^{2} \sin \xi_{1}} \sin \xi_{1} \frac{x}{l}-\frac{p l}{6 k} x^{3}+\left(\frac{1}{6}-\frac{1}{k}\right) \frac{p l^{3}}{k} x \\
& M_{c 2}(x)=-\frac{p l^{2}}{k \sinh \xi_{3}} \sinh \xi_{3} \frac{x}{l}+\frac{p l}{k} x \\
& E I \times w_{c 1}(x)=\frac{p l^{4}}{k^{2} \sinh \xi_{3}} \sinh \xi_{3} \frac{x}{l}-\frac{p l}{6 k} x^{3}+\left(\frac{1}{6}-\frac{1}{k}\right) \frac{p l^{3}}{k} x
\end{aligned}
$$

\section{- Non-uniform heating}

$$
\begin{gathered}
M_{c 1}(x)=A_{1} \cos \xi_{1} \frac{x}{l}+B_{1} \sin \xi_{1} \frac{x}{l}-E I \kappa_{T}, \quad E I \times w_{c 1}(x)=-A_{1} \frac{l^{2}}{k} \cos \xi_{1} \frac{x}{l}-B_{1} \frac{l^{2}}{k} \sin \xi_{1} \frac{x}{l}+A_{1} \frac{l^{2}}{k} \\
A_{1}=E I \kappa_{T} \quad B_{1}=E I \kappa_{T}\left(\frac{1}{\sin \xi_{1}}-\frac{1}{\tan \xi_{1}}\right)
\end{gathered}
$$

$$
\begin{gathered}
M_{c 2}(x)=A_{2} \cosh \xi_{3} \frac{x}{l}+B_{2} \sinh \xi_{3} \frac{x}{l}-E I \kappa_{T}, \quad E I \times w_{c 2}(x)=-A_{2} \frac{l^{2}}{k} \cosh \xi_{3} \frac{x}{l}-B_{2} \frac{l^{2}}{k} \sinh \xi_{3} \frac{x}{l}+A_{2} \frac{l^{2}}{k} \\
A_{2}=E I \kappa_{T} \quad B_{2}=E I \kappa_{T}\left(\frac{1}{\sinh \xi_{3}}-\frac{1}{\tanh \xi_{3}}\right)
\end{gathered}
$$




\section{Fixed-pinned beam}

\section{- Uniformly distributed load p}

$$
\begin{aligned}
& M_{c 1}(x)=A_{1} p l^{2} \cos \xi_{1} \frac{x}{l}+B_{1} p l^{2} \sin \xi_{1} \frac{x}{l}+\frac{p l^{2}}{k} \\
& E I \times w_{c 1}(x)=-A_{1} \frac{p l^{4}}{k} \cos \xi_{1} \frac{x}{l}-B_{1} \frac{p l^{4}}{k} \sin \xi_{1} \frac{x}{l}-\frac{p l^{2}}{2 k} x^{2}+\xi_{2} B_{1} \frac{p l^{3}}{k} x+A_{1} \frac{p l^{4}}{k} \\
& A_{1}=\frac{1 / 2 \sin \xi_{1}+\left(\xi_{2}-\sin \xi_{1}\right) / k}{\sin \xi_{1}-\xi_{2} \cos \xi_{1}} \quad B_{1}=\frac{(1 / k-1 / 2) \cos \xi_{1}-1 / k}{\sin \xi_{1}-\xi_{2} \cos \xi_{1}}
\end{aligned}
$$

$M_{c 2}(x)=A_{2} p l^{2} \cosh \xi_{3} \frac{x}{l}+B_{2} p l^{2} \sinh \xi_{3} \frac{x}{l}+\frac{p l^{2}}{k}$

$E I \times w_{c 2}(x)=-A_{2} \frac{p l^{4}}{k} \cosh \xi_{3} \frac{x}{l}-B_{2} \frac{p l^{4}}{k} \sinh \xi_{3} \frac{x}{l}-\frac{p l^{2}}{2 k} x^{2}+\xi_{4} B_{2} \frac{p l^{3}}{k} x+A_{2} \frac{p l^{4}}{k}$

$$
A_{2}=\frac{1 / 2 \sinh \xi_{3}+\left(\xi_{4}-\sinh \xi_{3}\right) / k}{\sinh \xi_{3}-\xi_{4} \cosh \xi_{3}} \quad B_{2}=\frac{(1 / k-1 / 2) \cosh \xi_{3}-1 / k}{\sinh \xi_{3}-\xi_{4} \cosh \xi_{3}}
$$

- Triangular distributed load (zero at $x=0$, and $p$ at $x=1)$

$M_{c 1}(x)=A_{1} p l^{2} \cos \xi_{1} \frac{x}{l}+B_{1} p l^{2} \sin \xi_{1} \frac{x}{l}+\frac{p l}{k} x$

$E I \times w_{c 1}(x)=-A_{1} \frac{p l^{4}}{k} \cos \xi_{1} \frac{x}{l}-B_{1} \frac{p l^{4}}{k} \sin \xi_{1} \frac{x}{l}-\frac{p l}{6 k} x^{3}+\left(B_{1} \xi_{2}+\alpha\right) \frac{p l^{3}}{k} x+A_{1} \frac{p l^{4}}{k}$

$$
A_{1}=\frac{(1 / 6-1 / k-\alpha) \sin \xi_{1}+\xi_{2} / k}{\sin \xi_{1}-\xi_{2} \cos \xi_{1}} \quad B_{1}=\frac{(1 / k+\alpha-1 / 6) \cos \xi_{1}-1 / k}{\sin \xi_{1}-\xi_{2} \cos \xi_{1}}
$$

$M_{c 2}(x)=A_{2} p l^{2} \cosh \xi_{3} \frac{x}{l}+B_{2} p l^{2} \sinh \xi_{3} \frac{x}{l}+\frac{p l}{k} x$

$E I \times w_{c 2}(x)=-A_{2} \frac{p l^{4}}{k} \cosh \xi_{3} \frac{x}{l}-B_{2} \frac{p l^{4}}{k} \sinh \xi_{3} \frac{x}{l}-\frac{p l}{6 k} x^{3}+\left(B_{2} \xi_{4}+\alpha\right) \frac{p l^{3}}{k} x+A_{2} \frac{p l^{4}}{k}$

$$
A_{2}=\frac{(1 / 6-1 / k-\alpha) \sinh \xi_{3}+\xi_{4} / k}{\sinh \xi_{3}-\xi_{4} \cosh \xi_{3}} \quad B_{2}=\frac{(1 / k+\alpha-1 / 6) \cosh \xi_{3}-1 / k}{\sinh \xi_{3}-\xi_{4} \cosh \xi_{3}}
$$


- Non-uniform heating

$$
\begin{aligned}
& M_{c 1}(x)=A_{1} \cos \xi_{1} \frac{x}{l}+B_{1} \sin \xi_{1} \frac{x}{l}-E I \kappa_{T} \\
& E I \times w_{c 1}(x)=-A_{1} \frac{l^{2}}{k} \cos \xi_{1} \frac{x}{l}-B_{1} \frac{l^{2}}{k} \sin \xi_{1} \frac{x}{l}+B_{1} \xi_{2} \frac{l}{k} x+A_{1} \frac{l^{2}}{k} \\
& A_{1}=E I \kappa_{T} \frac{\sin \xi_{1}-\xi_{2}}{\sin \xi_{1}-\xi_{2} \cos \xi_{1}} \quad B_{1}=E I \kappa_{T} \frac{1-\cos \xi_{1}}{\sin \xi_{1}-\xi_{2} \cos \xi_{1}}
\end{aligned}
$$

$$
\begin{aligned}
& M_{c 2}(x)=A_{2} \cosh \xi_{3} \frac{x}{l}+B_{2} \sinh \xi_{3} \frac{x}{l}-E I \kappa_{T} \\
& E I \times w_{c 2}(x)=-A_{2} \frac{l^{2}}{k} \cosh \xi_{3} \frac{x}{l}-B_{2} \frac{l^{2}}{k} \sinh \xi_{3} \frac{x}{l}+B_{2} \xi_{4} \frac{l}{k} x+A_{2} \frac{l^{2}}{k} \\
& A_{2}=E I \kappa_{T} \frac{\sinh \xi_{3}-\xi_{4}}{\sinh \xi_{3}-\xi_{4} \cosh \xi_{3}} \quad B_{2}=E I \kappa_{T} \frac{1-\cosh \xi_{3}}{\sinh \xi_{3}-\xi_{4} \cosh \xi_{3}}
\end{aligned}
$$

\section{Fixed-free beam}

\section{- Uniformly distributed load p}

$$
\begin{aligned}
& M_{c 1}(x)=A_{1} p l^{2} \cos \xi_{1} \frac{x}{l}+B_{1} p l^{2} \sin \xi_{1} \frac{x}{l}+\frac{p l^{2}}{k} \\
& E I \times w_{c 1}(x)=-A_{1} \frac{p l^{4}}{k} \cos \xi_{1} \frac{x}{l}-B_{1} \frac{p l^{4}}{k} \sin \xi_{1} \frac{x}{l}-\frac{p l^{2}}{2 k} x^{2}+\frac{p l^{3}}{k} x+A_{1} \frac{p l^{4}}{k} \\
& A_{1}=-\frac{\tan \xi_{1}}{\xi_{2}}-\frac{1}{k \cos \xi_{1}} \quad B_{1}=\frac{1}{\xi_{2}}
\end{aligned}
$$

$$
M_{c 2}(x)=A_{2} p l^{2} \cosh \xi_{3} \frac{x}{l}+B_{2} p l^{2} \sinh \xi_{3} \frac{x}{l}+\frac{p l^{2}}{k}
$$

$$
\begin{aligned}
& E I \times w_{c 2}(x)=-A_{2} \frac{p l^{4}}{k} \cosh \xi_{3} \frac{x}{l}-B_{2} \frac{p l^{4}}{k} \sinh \xi_{3} \frac{x}{l}-\frac{p l^{2}}{2 k} x^{2}+\frac{p l^{3}}{k} x+A_{2} \frac{p l^{4}}{k} \\
& A_{2}=-\frac{\tanh \xi_{3}}{\xi_{4}}-\frac{1}{k \cosh \xi_{3}} \quad B_{2}=\frac{1}{\xi_{4}}
\end{aligned}
$$


- $\quad$ Triangular distributed load (zero at $x=0$, and $p$ at $x=1)$

$$
\begin{aligned}
& M_{c 1}(x)=A_{1} p l^{2} \cos \xi_{1} \frac{x}{l}+B_{1} p l^{2} \sin \xi_{1} \frac{x}{l}+\frac{p l}{k} x \\
& E I \times w_{c 1}(x)=-A_{1} \frac{p l^{4}}{k} \cos \xi_{1} \frac{x}{l}-B_{1} \frac{p l^{4}}{k} \sin \xi_{1} \frac{x}{l}-\frac{p l}{6 k} x^{3}+\left(\xi_{2} B_{1}+\alpha\right) \frac{p l^{3}}{k} x+A_{1} \frac{p l^{4}}{k} \\
& A_{1}=-\frac{1}{k \cos \xi_{1}}-\frac{(1 / 2-1 / k-\alpha) \tan \xi_{1}}{\xi_{2}} \quad B_{1}=\frac{(1 / 2-1 / k-\alpha)}{\xi_{2}}
\end{aligned}
$$$$
M_{c 2}(x)=A_{2} p l^{2} \cosh \xi_{3} \frac{x}{l}+B_{2} p l^{2} \sinh \xi_{3} \frac{x}{l}+\frac{p l}{k} x
$$

$E I \times w_{c 2}(x)=-A_{2} \frac{p l^{4}}{k} \cosh \xi_{3} \frac{x}{l}-B_{2} \frac{p l^{4}}{k} \sinh \xi_{3} \frac{x}{l}-\frac{p l}{6 k} x^{3}+\left(\xi_{4} B_{2}+\alpha\right) \frac{p l^{3}}{k} x+A_{2} \frac{p l^{4}}{k}$

$$
A_{2}=-\frac{1}{k \cosh \xi_{3}}-\frac{(1 / 2-1 / k-\alpha) \tanh \xi_{3}}{\xi_{4}} \quad B_{2}=\frac{(1 / 2-1 / k-\alpha)}{\xi_{4}}
$$

- Non-uniform heating

$M_{c 1}(x)=E I \kappa_{T}\left(\frac{\cos \xi_{1} \frac{x}{l}}{\cos \xi_{1}}-1\right), \quad E I \times w_{c 1}(x)=E I \kappa_{T} \frac{l^{2}}{k \cos \xi_{1}}\left(1-\cos \xi_{1} \frac{x}{l}\right)$,

$$
M_{c 2}(x)=E I \kappa_{T}\left(\frac{\cosh \xi_{3} \frac{x}{l}}{\cosh \xi_{3}}-1\right), \quad E I \times w_{c 2}(x)=E I \kappa_{T} \frac{l^{2}}{k \cosh \xi_{3}}\left(1-\cosh \xi_{3} \frac{x}{l}\right),
$$




\section{Fixed-fixed beam}

\section{- Uniformly distributed load p}

$$
\begin{aligned}
& M_{c 1}(x)=A_{1} p l^{2} \cos \xi_{1} \frac{x}{l}+B_{1} p l^{2} \sin \xi_{1} \frac{x}{l}+\frac{p l^{2}}{k} \\
& E I \times w_{c 1}(x)=-A_{1} \frac{p l^{4}}{k} \cos \xi_{1} \frac{x}{l}-B_{1} \frac{p l^{4}}{k} \sin \xi_{1} \frac{x}{l}-\frac{p l^{2}}{2 k} x^{2}+\xi_{2} B_{1} \frac{p l^{3}}{k} x+A_{1} \frac{p l^{4}}{k} \\
& A_{1}=\frac{\left(1-\cos \xi_{1}\right) / 2-\left(\xi_{2}-\sin \xi_{1}\right) / \xi_{2}}{2-2 \cos \xi_{1}-\xi_{2} \sin \xi_{1}} \quad B_{1}=\frac{\left(1-\cos \xi_{1}\right) / \xi_{2}-1 / 2 \sin \xi_{1}}{2-2 \cos \xi_{1}-\xi_{2} \sin \xi_{1}}
\end{aligned}
$$

$$
\begin{aligned}
& M_{c 2}(x)=A_{2} p l^{2} \cosh \xi_{3} \frac{x}{l}+B_{2} p l^{2} \sinh \xi_{3} \frac{x}{l}+\frac{p l^{2}}{k} \\
& E I \times w_{c 2}(x)=-A_{2} \frac{p l^{4}}{k} \cosh \xi_{3} \frac{x}{l}-B_{2} \frac{p l^{4}}{k} \sinh \xi_{3} \frac{x}{l}-\frac{p l^{2}}{2 k} x^{2}+\xi_{4} B_{2} \frac{p l^{3}}{k} x+A_{2} \frac{p l^{4}}{k} \\
& A_{2}=\frac{\left(1-\cosh \xi_{3}\right) / 2-\left(\xi_{4}-\sinh \xi_{3}\right) / \xi_{4}}{2-2 \cosh \xi_{3}+\xi_{4} \sinh \xi_{3}} \quad B_{2}=\frac{\left(1-\cosh \xi_{3}\right) / \xi_{4}+1 / 2 \sinh \xi_{3}}{2-2 \cosh \xi_{3}+\xi_{4} \sinh \xi_{3}}
\end{aligned}
$$

\section{- Triangular distributed load (zero at $x=0$, and $p$ at $x=1$ )}

$M_{c 1}(x)=A_{1} p l^{2} \cos \xi_{1} \frac{x}{l}+B_{1} p l^{2} \sin \xi_{1} \frac{x}{l}+\frac{p l}{k} x$

$E I \times w_{c 1}(x)=-A_{1} \frac{p l^{4}}{k} \cos \xi_{1} \frac{x}{l}-B_{1} \frac{p l^{4}}{k} \sin \xi_{1} \frac{x}{l}-\frac{p l}{6 k} x^{3}+\left(\xi_{2} B_{1}+\alpha\right) \frac{p l^{3}}{k} x+A_{1} \frac{p l^{4}}{k}$

$A_{1}=\frac{(1 / 6-\alpha)\left(1-\cos \xi_{1}\right)-1 / 2\left(\xi_{2}-\sin \xi_{1}\right) / \xi_{2}}{2-2 \cos \xi_{1}-\xi_{2} \sin \xi_{1}}, \quad B_{1}=\frac{1 / 2\left(1-\cos \xi_{1}\right) / \xi_{2}-(1 / 6-\alpha) \sin \xi_{1}}{2-2 \cos \xi_{1}-\xi_{2} \sin \xi_{1}}$

$M_{c 2}(x)=A_{2} p l^{2} \cosh \xi_{3} \frac{x}{l}+B_{2} p l^{2} \sinh \xi_{3} \frac{x}{l}+\frac{p l}{k} x$

$E I \times w_{c 2}(x)=-A_{2} \frac{p l^{4}}{k} \cosh \xi_{3} \frac{x}{l}-B_{2} \frac{p l^{4}}{k} \sinh \xi_{3} \frac{x}{l}-\frac{p l}{6 k} x^{3}+\left(\xi_{4} B_{2}+\alpha\right) \frac{p l^{3}}{k} x+A_{2} \frac{p l^{4}}{k}$

$A_{2}=\frac{(1 / 6-\alpha)\left(1-\cosh \xi_{3}\right)-1 / 2\left(\xi_{4}-\sinh \xi_{3}\right) / \xi_{4}}{2-2 \cosh \xi_{3}+\xi_{4} \sinh \xi_{3}}, \quad B_{2}=\frac{1 / 2\left(1-\cosh \xi_{3}\right) / \xi_{4}+(1 / 6-\alpha) \sinh \xi_{3}}{2-2 \cosh \xi_{3}+\xi_{4} \sinh \xi_{3}}$ 
- Non-uniform heating

$$
\begin{array}{ll}
M_{c 1}(x)=-E I \kappa_{T} & E I \times w_{c 1}(x)=0 \\
M_{c 2}(x)=-E I \kappa_{T} & E I \times w_{c 2}(x)=0
\end{array}
$$

\section{Appendix F Timoshenko beams subjected to a concentrated load and an axial force}

The compressive forces are assumed smaller (absolute values) than the buckling load.

Equations (49) to (55) are applied in case of a compressive force with $k \alpha>-1$, and Equations (60) to (66) are applied in case of a tensile force or a compressive force with $k \alpha<-1$. In the case of a compressive force with $k \alpha>-1$, the constants of integration left of the concentrated force are $\mathrm{A}_{11}, \mathrm{~B}_{11}, \mathrm{NC}_{11}$, and $\mathrm{ND}_{11}$, and right of the concentrated force they are $\mathrm{A}_{12}, \mathrm{~B}_{12}, \mathrm{NC}_{12}$, and $\mathrm{ND}_{12}$. In the case of a tensile force or a compressive force with $\mathrm{k} \alpha<-1$, the constants of integration left of the concentrated force are $\mathrm{A}_{21}, \mathrm{~B}_{21}, \mathrm{NC}_{21}$, and $\mathrm{ND}_{21}$, and right of the concentrated force they are $\mathrm{A}_{22}$, $\mathrm{B}_{22}, \mathrm{NC}_{22}$, and $\mathrm{ND}_{22}$.

\section{Fixed-pinned beam}

Case of a compressive force with $k \alpha>-1$ : following boundary conditions and continuity conditions are applied:

$$
\begin{array}{lll}
\checkmark \quad \mathrm{w}\left(\mathrm{x}_{1}=0\right)=0: & -\mathrm{A}_{11}+\mathrm{ND}_{11}=0 \\
\checkmark & \varphi\left(\mathrm{x}_{1}=0\right)=0: & -\mathrm{B}_{11} \xi_{2}+\mathrm{NC}_{11} \times 1=0 \\
\checkmark & \mathrm{w}\left(\mathrm{x}_{1}=\mathrm{a}\right)=\mathrm{w}\left(\mathrm{x}_{2}=0\right): & -\mathrm{A}_{11} \cos \xi_{1} \mathrm{a} / 1-\mathrm{B}_{11} \sin \xi_{1} \mathrm{a} / 1+\mathrm{NC}_{11} \times \mathrm{a}+\mathrm{ND}_{11}=-\mathrm{A}_{12}+\mathrm{ND}_{12} \\
\checkmark \quad \varphi\left(\mathrm{x}_{1}=\mathrm{a}\right)=\varphi\left(\mathrm{x}_{2}=0\right): & \mathrm{A}_{11} \xi_{2} \sin \xi_{1} \mathrm{a} / 1-\mathrm{B}_{11} \xi_{2} \cos \xi_{1} \mathrm{a} / 1+\mathrm{NC}_{11} \times 1=-\mathrm{B}_{12} \xi_{2}+\mathrm{NC}_{12} \times 1 \\
\checkmark \quad \mathrm{M}\left(\mathrm{x}_{1}=\mathrm{a}\right)=\mathrm{M}\left(\mathrm{x}_{2}=0\right): & \mathrm{A}_{11} \cos \xi_{1} \mathrm{a} / 1+\mathrm{B}_{11} \sin \xi_{1} \mathrm{a} / 1=\mathrm{A}_{12} \\
\checkmark \quad \mathrm{T}\left(\mathrm{x}_{1}=\mathrm{a}\right)-\mathrm{T}\left(\mathrm{x}_{2}=0\right)=\mathrm{P}: & \mathrm{NC}_{11}-\mathrm{NC}_{12}=\mathrm{P} \\
\checkmark \quad \mathrm{M}\left(\mathrm{x}_{2}=\mathrm{b}\right)=0: & \mathrm{A}_{12} \cos \xi_{1} \mathrm{~b} / 1+\mathrm{B}_{12} \sin \xi_{1} \mathrm{~b} / 1=0 \\
\checkmark \quad \mathrm{w}\left(\mathrm{x}_{2}=\mathrm{b}\right)=0: & -\mathrm{A}_{12} \cos \xi_{1} \mathrm{~b} / 1-\mathrm{B}_{12} \sin \xi_{1} \mathrm{~b} / 1+\mathrm{NC}_{12} \times \mathrm{b}+\mathrm{ND}_{12}=0
\end{array}
$$


Solving the equation system yields the constants of integration as follows:

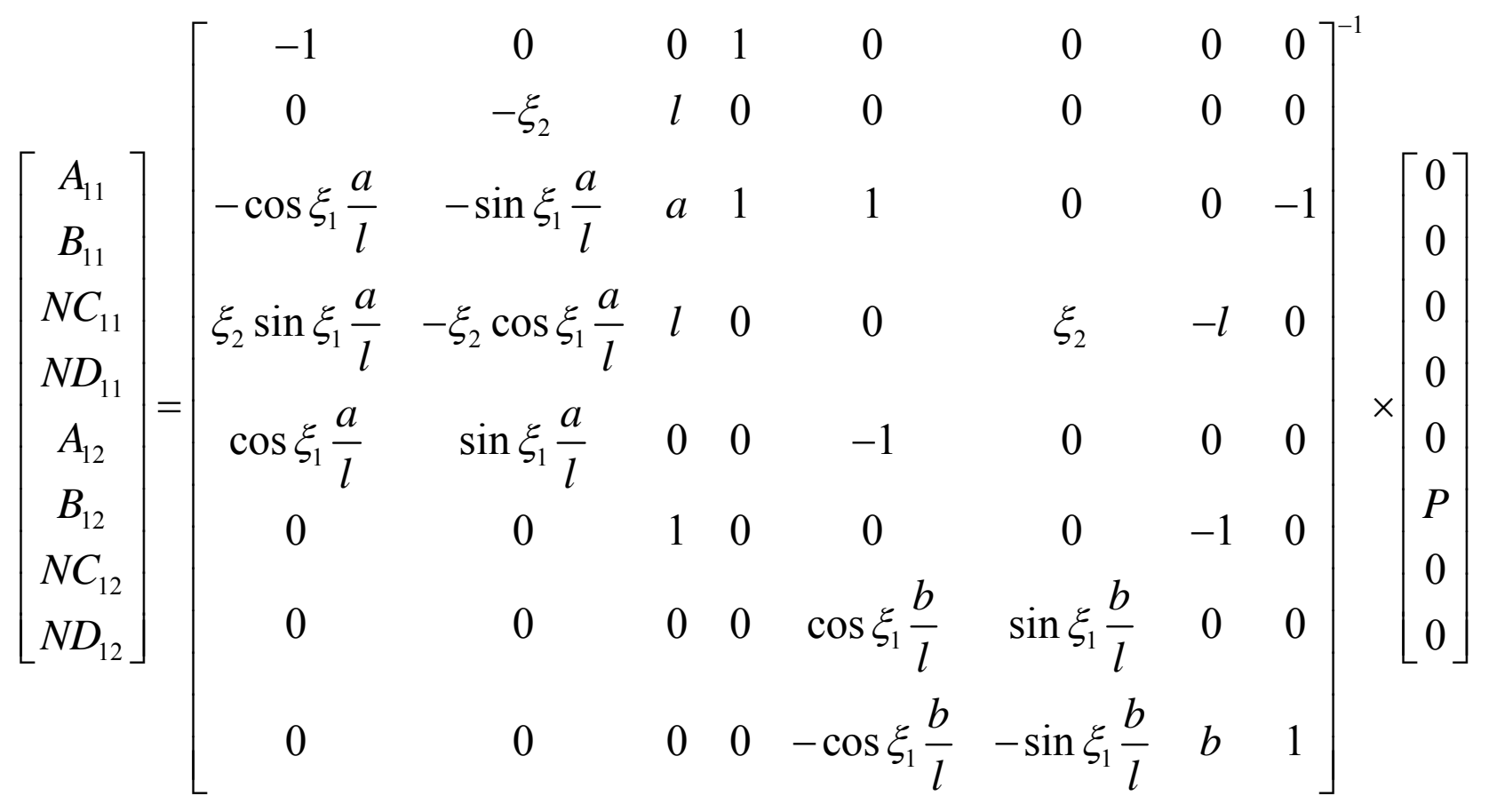

Case of a tensile force or a compressive force with $k \alpha<-1$ : the corresponding boundary conditions and continuity conditions are applied. Solving the equation system yields the constants of integration as follows:

$\left[\begin{array}{c}A_{21} \\ B_{21} \\ N C_{21} \\ N D_{21} \\ A_{22} \\ B_{22} \\ N C_{22} \\ N D_{22}\end{array}\right]=\left[\begin{array}{cccccccc}-1 & 0 & 0 & 1 & 0 & 0 & 0 & 0 \\ 0 & -\xi_{4} & l & 0 & 0 & 0 & 0 & 0 \\ -\cosh \xi_{3} \frac{a}{l} & -\sinh \xi_{3} \frac{a}{l} & a & 1 & 1 & 0 & 0 & -1 \\ -\xi_{4} \sinh \xi_{3} \frac{a}{l} & -\xi_{4} \cosh \xi_{3} \frac{a}{l} & l & 0 & 0 & \xi_{4} & -l & 0 \\ \cosh \xi_{3} \frac{a}{l} & \sinh \xi_{3} \frac{a}{l} & 0 & 0 & -1 & 0 & 0 & 0 \\ 0 & 0 & 1 & 0 & 0 & 0 & -1 & 0 \\ 0 & 0 & 0 & 0 & \cosh \xi_{3} \frac{b}{l} & \sinh \xi_{3} \frac{b}{l} & 0 & 0 \\ 0 & 0 & 0 & 0 & -\cosh \xi_{3} \frac{b}{l} & -\sinh \xi_{3} \frac{b}{l} & b & 1\end{array}\right]_{0}^{-1} \times\left[\begin{array}{l}0 \\ 0 \\ 0 \\ 0 \\ 0 \\ 0 \\ 0\end{array}\right]$




\section{Pinned-pinned beam}

Case of a compressive force with $k \alpha>-1$

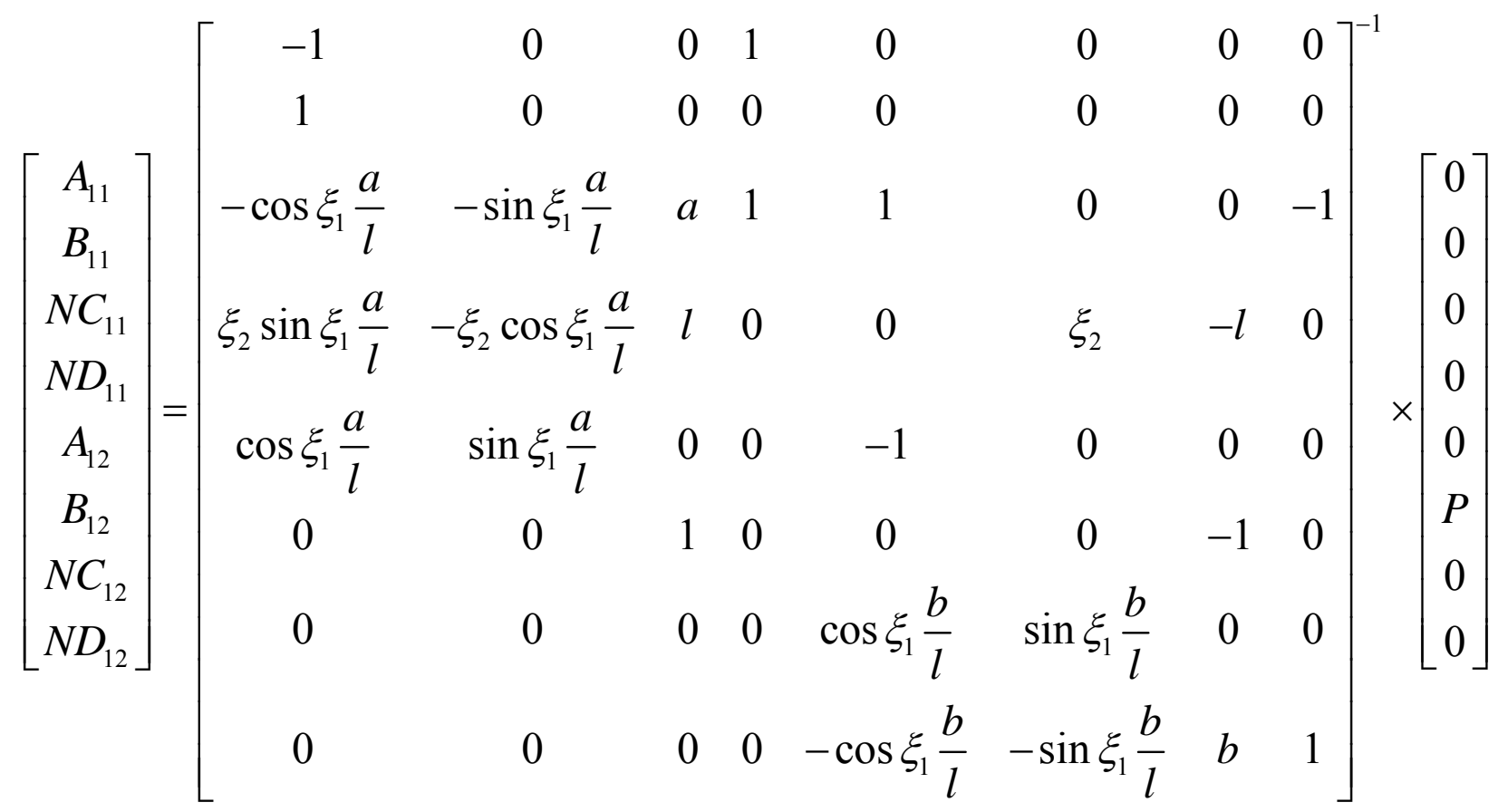

Case of a tensile force or a compressive force with $\mathrm{k} \alpha<-1$

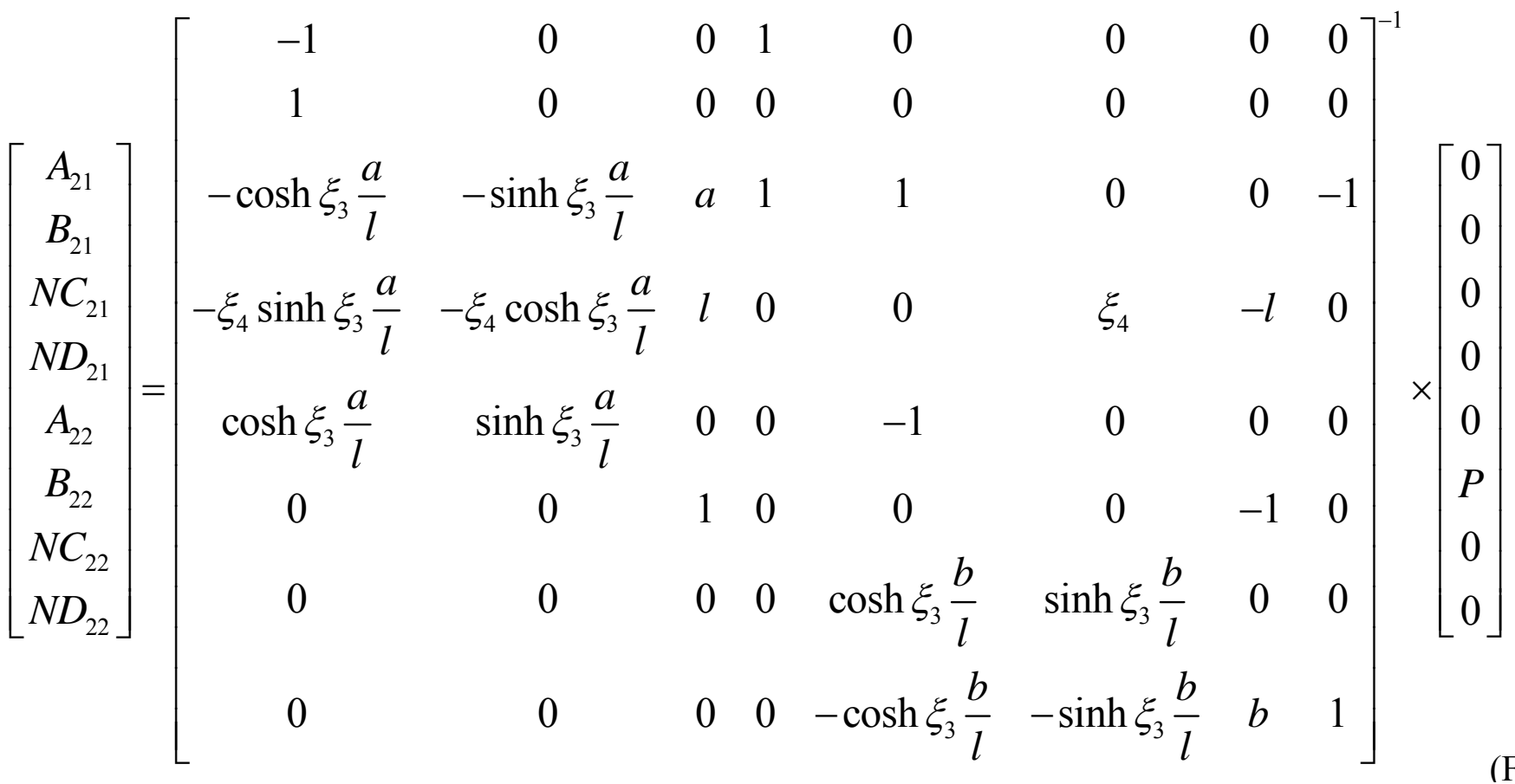




\section{Fixed-fixed beam}

Case of a compressive force with $k \alpha>-1$

$\left[\begin{array}{c}A_{11} \\ B_{11} \\ N C_{11} \\ N D_{11} \\ A_{12} \\ B_{12} \\ N C_{12} \\ N D_{12}\end{array}\right]=\left[\begin{array}{cccccccc}-1 & 0 & 0 & 1 & 0 & 0 & 0 & 0 \\ 0 & -\xi_{2} & l & 0 & 0 & 0 & 0 & 0 \\ -\cos \xi_{1} \frac{a}{l} & -\sin \xi_{1} \frac{a}{l} & a & 1 & 1 & 0 & 0 & -1 \\ \xi_{2} \sin \xi_{1} \frac{a}{l} & -\xi_{2} \cos \xi_{1} \frac{a}{l} & l & 0 & 0 & \xi_{2} & -l & 0 \\ \cos \xi_{1} \frac{a}{l} & \sin \xi_{1} \frac{a}{l} & 0 & 0 & -1 & 0 & 0 & 0 \\ 0 & 0 & 1 & 0 & 0 & 0 & -1 & 0 \\ 0 & 0 & 0 & 0 & \xi_{2} \sin \xi_{1} \frac{b}{l} & -\xi_{2} \cos \xi_{1} \frac{b}{l} & l & 0 \\ 0 & 0 & 0 & 0 & -\cos \xi_{1} \frac{b}{l} & -\sin \xi_{1} \frac{b}{l} & b & 1\end{array}\right]^{-1} \times\left[\begin{array}{l}0 \\ 0 \\ 0 \\ 0 \\ 0 \\ 0 \\ 0 \\ 0\end{array}\right]$

(F6)

Case of a tensile force or a compressive force with $\mathrm{k} \alpha<-1$

\begin{tabular}{|c|c|c|c|c|c|c|c|c|c|c|}
\hline & & -1 & 0 & 0 & 1 & 0 & 0 & 0 & $0^{-}$ & \\
\hline & & 0 & $-\xi_{4}$ & $l$ & 0 & 0 & 0 & 0 & 0 & \\
\hline $\begin{array}{l}A_{21} \\
B_{21}\end{array}$ & & $-\cosh \xi_{3} \frac{a}{l}$ & $-\sinh \xi_{3} \frac{a}{l}$ & $a$ & 1 & 1 & 0 & 0 & -1 & $\left.\begin{array}{l}0 \\
0\end{array}\right]$ \\
\hline $\begin{array}{l}N C_{21} \\
N D_{21}\end{array}$ & & $-\xi_{4} \sinh \xi_{3} \frac{a}{l}$ & $-\xi_{4} \cosh \xi_{3} \frac{a}{l}$ & $l$ & 0 & 0 & $\xi_{4}$ & $-l$ & 0 & $\begin{array}{l}0 \\
0\end{array}$ \\
\hline$A_{22}$ & & $\cosh \xi_{3} \frac{a}{l}$ & $\sinh \xi_{3} \frac{a}{l}$ & 0 & 0 & -1 & 0 & 0 & 0 & \begin{tabular}{l|l}
$\times$ & 0
\end{tabular} \\
\hline$B_{22}$ & & 0 & 0 & 1 & 0 & 0 & 0 & -1 & 0 & $P$ \\
\hline$N C_{22}$ & & & & & & 6. & & & & 0 \\
\hline$\left.-N D_{22}\right]$ & & 0 & 0 & 0 & 0 & $-\xi_{4} \sinh \xi_{3} \frac{}{l}$ & $-\xi_{4} \cosh \xi_{3} \bar{l}$ & I & 0 & 0 \\
\hline & & 0 & 0 & 0 & 0 & $-\cosh \xi_{3} \frac{b}{l}$ & $-\sinh \xi_{3} \frac{b}{l}$ & $b$ & 1 & \\
\hline
\end{tabular}




\section{Fixed-free beam}

Case of a compressive force with $\mathrm{k} \alpha>-1$

$$
\left.\left[\begin{array}{c}
A_{11} \\
B_{11} \\
N C_{11} \\
N D_{11} \\
A_{12} \\
B_{12} \\
N C_{12} \\
N D_{12}
\end{array}\right]=\left[\begin{array}{cccccccc}
-1 & 0 & 0 & 1 & 0 & 0 & 0 & 0 \\
0 & -\xi_{2} & l & 0 & 0 & 0 & 0 & 0 \\
-\cos \xi_{1} \frac{a}{l} & -\sin \xi_{1} \frac{a}{l} & a & 1 & 1 & 0 & 0 & -1 \\
\xi_{2} \sin \xi_{1} \frac{a}{l} & -\xi_{2} \cos \xi_{1} \frac{a}{l} & l & 0 & 0 & \xi_{2} & -l & 0 \\
\cos \xi_{1} \frac{a}{l} & \sin \xi_{1} \frac{a}{l} & 0 & 0 & -1 & 0 & 0 & 0 \\
0 & 0 & 1 & 0 & 0 & 0 & -1 & 0 \\
0 & 0 & 0 & 0 & \cos \xi_{1} \frac{b}{l} & \sin \xi_{1} \frac{b}{l} & 0 & 0 \\
0 & 0 & 0 & 0 & 0 & 0 & 1 & 0
\end{array}\right]^{-1} \times \begin{array}{l}
0 \\
0 \\
0 \\
0 \\
0 \\
0 \\
0
\end{array}\right]
$$

Case of a tensile force or a compressive force with $\mathrm{k} \alpha<-1$ :

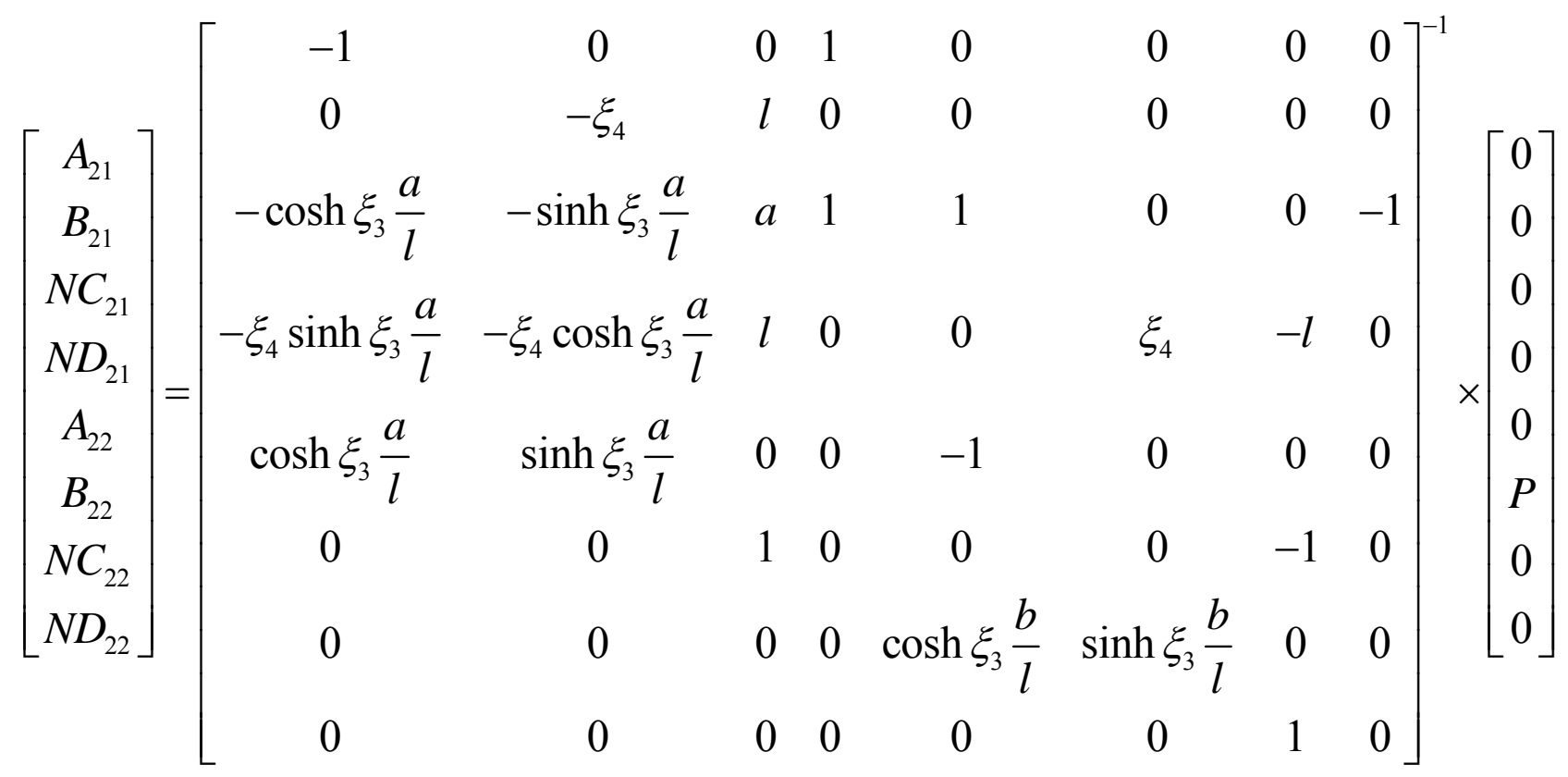


For a concentrated load P applied at the tip of the fixed-free beam, the moment and deflection curves are as follows:

$$
\begin{aligned}
& M_{c 1}(x)=-P l \frac{\tan \xi_{1}}{\xi_{2}} \cos \xi_{1} \frac{x}{l}+\frac{P l}{\xi_{2}} \sin \xi_{1} \frac{x}{l} \\
& E I \times w_{c 1}(x)=\frac{P l^{3}}{k} \frac{\tan \xi_{1}}{\xi_{2}} \cos \xi_{1} \frac{x}{l}-\frac{P l^{3}}{k \xi_{2}} \sin \xi_{1} \frac{x}{l}+\frac{P l^{2}}{k} x-\frac{P l^{3}}{k} \frac{\tan \xi_{1}}{\xi_{2}} \\
& M_{c 2}(x)=-P l \frac{\tanh \xi_{3}}{\xi_{4}} \cosh \xi_{3} \frac{x}{l}+\frac{P l}{\xi_{4}} \sinh \xi_{3} \frac{x}{l} \\
& E I \times w_{c 2}(x)=\frac{P l^{3}}{k} \frac{\tanh \xi_{3}}{\xi_{4}} \cosh \xi_{3} \frac{x}{l}-\frac{P l^{3}}{k \xi_{4}} \sinh \xi_{3} \frac{x}{l}+\frac{P l^{2}}{k} x-\frac{P l^{3}}{k} \frac{\tanh \xi_{3}}{\xi_{4}}
\end{aligned}
$$

It is worth mentioning that the results of Equation (F10) satisfy the following equations of equilibrium using the free body diagram:

$$
\begin{aligned}
& M_{c 1}(x=0)+P l-N w_{c 1}(x=l)=0 \\
& M_{c 2}(x=0)+P l-N w_{c 2}(x=l)=0
\end{aligned}
$$

For the case of an external moment $\mathrm{M}^{*}$ (in counterclockwise) applied at the beam the continuity equations are modified as follows:

Case of a compressive force with $k \alpha>-1$

$$
\begin{array}{lll}
\checkmark & \mathrm{M}\left(\mathrm{x}_{1}=\mathrm{a}\right)-\mathrm{M}\left(\mathrm{x}_{2}=0\right)=\mathrm{M}^{*} & \mathrm{~A}_{11} \cos \xi_{1} \mathrm{a} / 1+\mathrm{B}_{11} \sin \xi_{1} \mathrm{a} / 1-\mathrm{A}_{12}=\mathrm{M}^{*} \\
\checkmark & \mathrm{T}\left(\mathrm{x}_{1}=\mathrm{a}\right)-\mathrm{T}\left(\mathrm{x}_{2}=0\right)=0: & \mathrm{NC}_{11}-\mathrm{NC}_{12}=0
\end{array}
$$

Case of a tensile force or a compressive force with $\mathrm{k} \alpha<-1$

$$
\begin{array}{lll}
\checkmark & \mathrm{M}\left(\mathrm{x}_{1}=\mathrm{a}\right)=\mathrm{M}\left(\mathrm{x}_{2}=0\right): & \mathrm{A}_{21} \cosh \xi_{3} \mathrm{a} / 1+\mathrm{B}_{21} \sinh \xi_{3} \mathrm{a} / 1-\mathrm{A}_{2}=\mathrm{M}^{*} \\
\checkmark & \mathrm{T}\left(\mathrm{x}_{1}=\mathrm{a}\right)-\mathrm{T}\left(\mathrm{x}_{2}=0\right)=0: & \mathrm{NC}_{21}-\mathrm{NC}_{22}=0
\end{array}
$$




\section{TIMOSHENKO BEAM THEORY EXACT SOLUTION}

\section{References}

Abbas, M-O. and Mohammad, K. 2013. Finite element formulation for stability and free vibration analysis of Timoshenko beam. Advances in Acoustics and Vibration, vol 2013. https://doi.org/10.1155/2013/841215

Chen, Y., Dong, S., Zang, Z., Zhang, Q., Zhang, J., Liu, X., Zhang, H., Lou, F. and Ao, C. 2018. Variational Iteration Approach for Flexural Vibration of Rotating Timoshenko Cantilever Beams. International Journal of Structural Stability and Dynamics, Vol. 18, No. 12, 1850154 (2018). https://doi.org/10.1142/S0219455418501547

Hayrullah, G.K. and Mustapha, O.Y. 2017. Buckling analysis of non-local Timoshenko beams by using Fourier series. Int J Eng Appl Sci, vol. 9(4), 89-99. https://doi.org/10.24107/ijeas.362242

Hu, Z.P., Pan, W.H. and Tong, J.Z. 2019. Exact solutions for buckling and second-order effect of shear deformable Timoshenko beam-columns based on matrix structural analysis. Appl. Sci. 2019, 9(18), 3814. https://doi.org/10.3390/app9183814

Onyia, M.E. and Rowland-Lato, E.O. 2018. Determination of the critical buckling load of shear deformable unified beam. IJET, Vol 10 No 3. DOI: 10.21817/ijet/2018/v10i3/181003026

Pavlovic, R. and Pavlović, I. 2018. Dynamic stability of Timoshenko beams on Pasternak viscoelastic foundation. Theoretical and Applied Mechanics 45(00):5-5, 2018. DOI:10.2298/TAM171103005P

Sang-Ho, K., Sun-Jin, H. and Kang, S.K. 2019. Nonlinear finite element analysis formulation for shear in reinforced concrete beams. Appl. Sci. 2019, 9, 3503. https://doi.org/10.3390/app9173503

Timoshenko, S.P. 1921. On the correction for shear of the differential equation for transverse vibrations of prismatic bar. Philos Mag 1921; 41: 744-746.

Timoshenko, S.P. and Gere, J.M. 1961. Theory of Elastic Stability; McGraw-Hill: New York, USA, 1961. 